\title{
Current Biology \\ Callose-Regulated Symplastic Communication Coordinates Symbiotic Root Nodule Development
}

\section{Graphical Abstract}

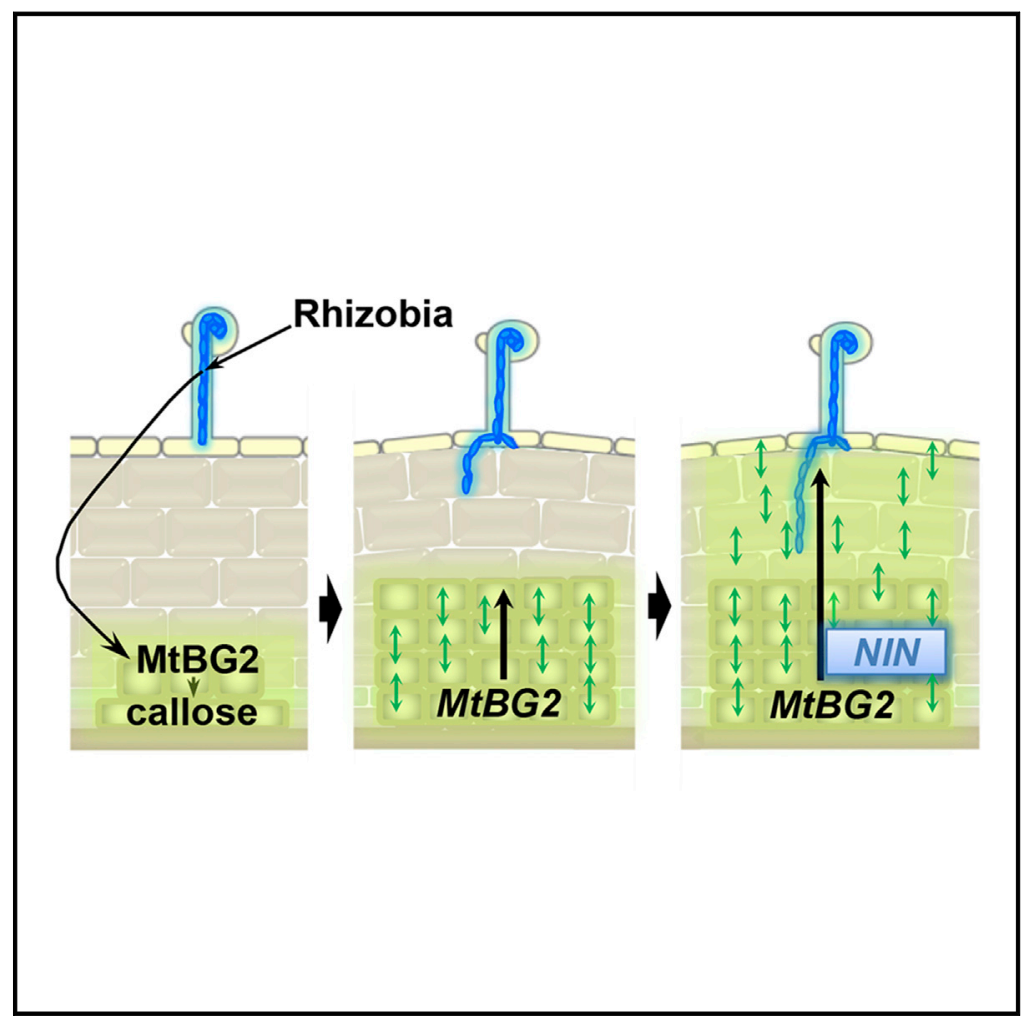

Highlights

- Rhizobia modify $\beta$-1,3-glucan (callose) levels in developing nitrogen-fixing nodules

- The $\beta-1,3$-glucanase MtBG2 promotes symplastic connectivity in nodule primordia

- Symplastic communication is necessary for nodule development and colonization

- Symplastic connectivity regulates the spatial expression of key symbiotic genes

\section{Authors}

Rocio Gaudioso-Pedraza, Martina Beck, Lisa Frances, ..., Giles E.D. Oldroyd, Yoselin Benitez-Alfonso, Fernanda de Carvalho-Niebel

\section{Correspondence}

y.benitez-alfonso@leeds.ac.uk (Y.B.-A.), fernanda.de-carvalho-niebel@inra.fr (F.d.C.-N.)

\section{In Brief}

Gaudioso-Pedraza et al. show that symplastic communication regulates the formation of nitrogen-fixing nodules. Rhizobia-induced callose ( $\beta$-1,3-glucan) turnover, which is orchestrated by the $\beta$-1,3-glucanase MtBG2, promotes cellular connectivity required for the expression of key regulators and for coordinating organ development and colonization. 


\title{
Callose-Regulated Symplastic Communication Coordinates Symbiotic Root Nodule Development
}

\author{
Rocio Gaudioso-Pedraza, ${ }^{1,4}$ Martina Beck, ${ }^{2,4}$ Lisa Frances,,${ }^{2,4}$ Philip Kirk, ${ }^{1}$ Carolina Ripodas, ${ }^{2}$ Andreas Niebel, ${ }^{2}$ \\ Giles E.D. Oldroyd, ${ }^{3}$ Yoselin Benitez-Alfonso, ${ }^{1,4,{ }^{*}}$ and Fernanda de Carvalho-Niebel ${ }^{2,4,5, *}$ \\ ${ }^{1}$ Centre for Plant Sciences, School of Biology, University of Leeds, Leeds LS2 9JT, UK \\ ${ }^{2}$ LIPM, Université de Toulouse, INRA, CNRS, 31326 Castanet-Tolosan, France \\ ${ }^{3}$ Sainsbury Laboratory, Cambridge University, Bateman Street, Cambridge CB2 1LR, UK \\ ${ }^{4}$ These authors contributed equally \\ ${ }^{5}$ Lead Contact \\ *Correspondence: y.benitez-alfonso@leeds.ac.uk (Y.B.-A.), fernanda.de-carvalho-niebel@inra.fr (F.d.C.-N.) \\ https://doi.org/10.1016/j.cub.2018.09.031
}

\section{SUMMARY}

The formation of nitrogen-fixing nodules in legumes involves the initiation of synchronized programs in the root epidermis and cortex to allow rhizobial infection and nodule development. In this study, we provide evidence that symplastic communication, regulated by callose turnover at plasmodesmata (PD), is important for coordinating nodule development and infection in Medicago truncatula. Here, we show that rhizobia promote a reduction in callose levels in inner tissues where nodules initiate. This downregulation coincides with the localized expression of $M$. truncatula $\beta-1,3-$ glucanase 2 (MtBG2), encoding a novel PD-associated callose-degrading enzyme. Spatiotemporal analyses revealed that MtBG2 expression expands from dividing nodule initials to rhizobia-colonized cortical and epidermal tissues. As shown by the transport of fluorescent molecules in vivo, symplastic-connected domains are created in rhizobia-colonized tissues and enhanced in roots constitutively expressing MtBG2. MtBG2-overexpressing roots additionally displayed reduced levels of PD-associated callose. Together, these findings suggest an active role for MtBG2 in callose degradation and in the formation of symplastic domains during sequential nodule developmental stages. Interfering with symplastic connectivity led to drastic nodulation phenotypes. Roots ectopically expressing $\beta-1,3$-glucanases (including MtBG2) exhibited increased nodule number, and those expressing MtBG2 RNAi constructs or a hyperactive callose synthase (under symbiotic promoters) showed defective nodulation phenotypes. Obstructing symplastic connectivity appears to block a signaling pathway required for the expression of NODULE INCEPTION (NIN) and its target NUCLEAR FACTOR-YA1 (NF-YA1) in the cortex. We conclude that symplastic intercellular communication is proactively enhanced by rhizobia, and this is necessary for appropriate coordination of bacterial infection and nodule development.

\section{INTRODUCTION}

Legumes establish symbiotic associations with nitrogen-fixing rhizobia, which, hosted within newly formed root nodules, have the optimized environment for fixing atmospheric dinitrogen for the plant [1]. Nodule formation involves coordinated reprogramming at different tissues to allow intracellular bacterial entry and the activation of the nodule organogenesis program. Endosymbiotic entry occurs via newly formed tubular structures, initiated from bacterial-entrapped root hairs, called infection threads (ITs) [2]. This host-constructed transcellular compartment guides rhizobia to the underlying nodule primordium in the cortex, where the bacteria are released and differentiate into nitrogen-fixing bacteroids. Although infection and organogenesis can be genetically separated, they need to be spatiotemporally coordinated for the successful formation of a nitrogen-fixing nodule [3]. Nodule development engages local hormonal changes and is tightly controlled by a systemic autoregulatory pathway (AON) that restricts nodule number [4]. Although diffusible hormones, such as cytokinin and auxin [5, 6], are important for initiating nodule organogenesis, little is known of how signal transmission is regulated from the infection site to underlying root tissues to coordinate nodule development.

The host plant perceives rhizobia-secreted lipo-chitooligosaccharide nodulation factors (NFs) via an ancient signal transduction system, which is partly shared during the establishment of the arbuscular mycorrhizal (AM) symbiosis [7]. Calcium oscillations are a common component of this pathway, decoded in the nucleus by the calcium- and calmodulin-dependent protein kinase CCaMK $[8,9]$. CCaMK interacts with and phosphorylates the transcriptional regulator CYCLOPS that orchestrates the downstream expression of nodule inception (NIN) during nodulation $[10,11]$ and ETHYLENE RESPONSE FACTOR (ERF) REQUIRED FOR MODULATION 1 (ERN1) [12, 13], which are themselves also regulated by other transcription factors or regulatory complexes [14-20]. Both ERN1 (with its homolog ERN2) and NIN are crucial for the construction of root hair infection threads, where they individually regulate the transcription of cell-wall-associated ENOD11/12 and NODULATION PECTATE LYASE 1 (NPL1) genes, respectively [2, 10-14, 21-24]. These 

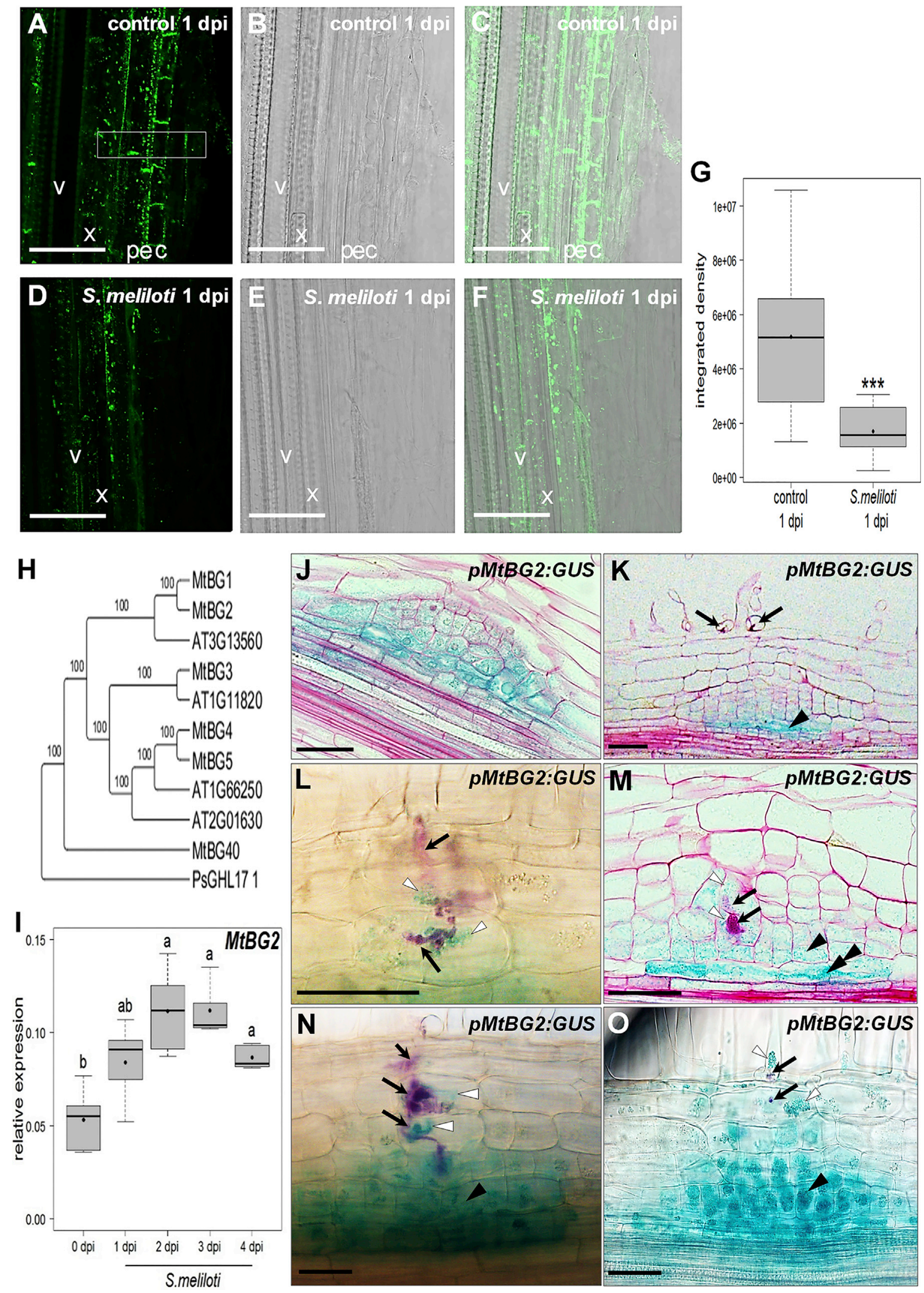

S. mellioti $1 \mathrm{dpi}$
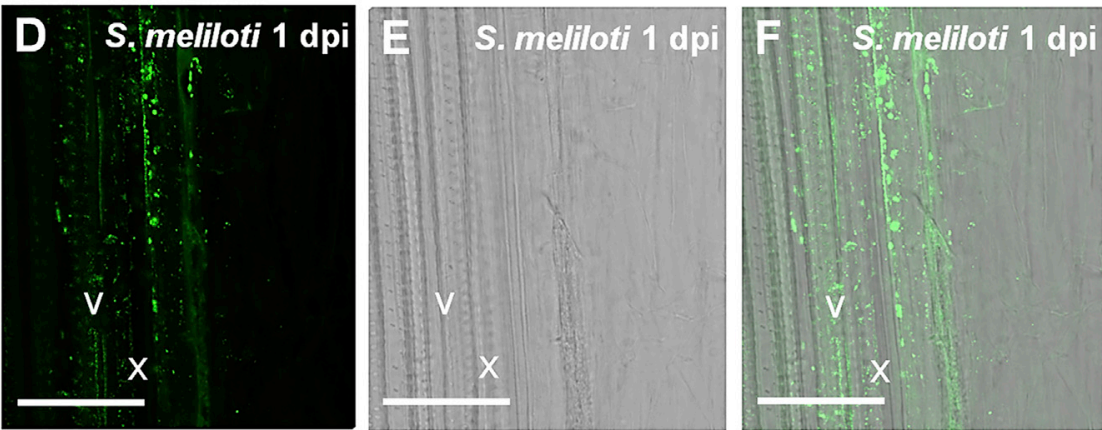

(legend on next page) 
and other infection-related regulators are also required for nodule organogenesis in the cortex [10, 11, 16, 22, 23, 25], underlying their potential roles in the infection-organogenesis signaling crosstalk. NIN has emerged as a central evolutionary-conserved nodulation-associated regulator [26, 27] sufficient to trigger organogenesis in the root cortex, where it regulates the expression of NUCLEAR FACTOR-Y (NF-Y) and EPR3 genes [28-30]. $N I N$ is also involved in the negative feedback regulation of infection-related responses $[30,31]$ and in the long-distance regulation of nodulation via the activation of CLAVATA/EMBRYO SURROUNDING REGION (CLE) peptide-encoding genes [32, 33], and when ectopically expressed in the root epidermis, it triggers organogenesis in the cortex [30]. These findings place NIN in a central position in the regulation of both cell-autonomous and non-cell-autonomous symbiotic signaling, but how these functions are coordinated remains largely unknown.

Past research revealed that symplastic connections are established between the phloem and nodule initials [34], raising the question of whether intercellular trafficking via plasmodesmata (PD) [35], channels connecting the cytoplasm and endoplasmic reticulum (ER) of neighboring cells, might contribute to signaling during nodulation. The epidermal cells undergoing infection must communicate with cortical and pericycle cells where division is initiated [3] to form the nodule primordia. These processes are tightly integrated, and organogenesis ceases if infection aborts. Such integration suggests the potential need for symplastic transport pathways, which allows the transport of molecules (such as proteins and RNAs) to coordinate signaling and enable rapid responses.

A major regulator for symplastic communication is the cell wall $\beta-1,3$ glucan, callose. Callose turnover at PD sites involves the localized action of callose synthases (CALSs) and $\beta-1,3$-glucanases (BGs) [36]. In this study, we provide evidence for the importance of symplastic cell-to-cell communication during nodulation. We show that callose is reduced in root tissues form- ing the primordia and that this correlates with the progressive expression of a rhizobia-induced, PD-located, callose-degrading enzyme. We further demonstrate that these changes in callose degradation correlate with increased symplastic connectivity in rhizobia-colonized tissues. The selective modification of callose levels at PD sites by ectopic expression of $\beta-1,3$ glucanases [36] or by expressing RNAi constructs or a hyperactive CALS3 version (cals3m) [37] led to changes in symplastic connectivity and strong nodulation phenotypes, associated with the deregulated expression of key symbiotic regulators. Here, we propose a model highlighting the importance of callosedegrading enzymes for the creation of symplastic domains, enabling the transport of yet unknown mobile signal(s) that coordinate nodule developmental programs.

\section{RESULTS}

Rhizobial Inoculation Modulates Callose Levels and the Early Expression of Callose-Degrading $\beta$-1,3Glucanases in M. truncatula Roots

Past research indicates that symplastic connectivity increases between phloem and nodule initials (from pericycle to inner cortical cells) as early as $48 \mathrm{hr}$ upon inoculation with rhizobia [34], raising the question of whether callose regulation is involved in this process. To test this, we monitored callose levels in $M$. truncatula roots before and $24 \mathrm{hr}$ after inoculation with rhizobia. Immunolocalization of callose was carried out in root sections 1 day after spot inoculation (1 dpi) with either water (mock control) or with a rhizobia bacterial suspension (Sinorhizobium meliloti). Strong callose labeling was detected as punctate sites, indicative of PDs, in inner root tissues (cortex, pericycle, and endodermis) of mock control roots (Figures 1A-1C). Punctate labeling was also observed in rhizobia-inoculated roots (Figures 1D-1F), but the fluorescence signal in the inner tissues was reduced by comparison to the mock-inoculated control.

Figure 1. Decreased Callose Levels and Induced Expression of $\beta-1,3-$ Glucanases in M. truncatula Roots Inoculated with Rhizobia (A-F) Immunolocalization reveals a downregulation of callose levels in roots spot inoculated with $S$. meliloti. Callose was detected using a monoclonal antibody and Alexa-488-conjugated secondary antibody in longitudinal sections of $M$. truncatula roots 1 day post spot-inoculation with mock water control (mock 1 dpi; A-C) or with a S. meliloti bacterial suspension (S. meliloti $1 \mathrm{dpi}$; D-F). Transmission light sections (B and E) are shown for signal co-localization (C and F) in the vascular cylinder (v), xylem (x), pericycle (p), endodermis (e), and cortex (c).

(G) Green fluorescence (callose signal) was compared by calculating the integrated density (arbitary units [a.u.]; measured using ImageJ) for each image (14 images/sample) from at least three biological repetitions, using a region of interest (indicated in A) of approximately $1,000 \mu \mathrm{m}^{2}$ that excluded the vascular and xylem tissues. An Aspin-Welch two-sample t test was performed in R (asterisks indicate statistical difference compared with mock control; ${ }^{\star \star \star} \mathrm{p}<0.001$ ).

$(H)$ A phylogenetic tree depicting the relationship between a set of $M$. truncatula and $A$. thaliana $\beta-1,3-g$ lucanases. Numbers correspond to Bayesian posterior probabilities. The sequence of a predicted $\beta-1,3-$ glucanase from Picea sitchensis (Ps GHL17_1) was included as an outgroup.

(I) qRT-PCR revealed upregulation of Medtr3g083580 (MtBG2 in I) expression in total RNA samples extracted from $M$. truncatula roots before (0 dpi) and after (1-4 dpi) inoculation with rhizobia. Boxplots represent average values from individual pooled samples $(n=100-125 /$ sample) from five independent biological experiments after normalization against three reference transcripts. One-way ANOVA followed by a Tukey honest significant difference (HSD) test of the values were performed in $\mathrm{R}(\mathrm{p}<0.001)$. Classes sharing the same letter are not significantly different.

(J-O) Representative images of $M$. truncatula roots expressing a pMtBG2:GUS fusion construct. GUS activity appears in blue, and counterstaining with Ruthenium Red in $(\mathrm{J}),(\mathrm{K})$, and $(\mathrm{M})$ reveals cell outlines. In non-inoculated conditions, GUS staining (in blue) is restricted to lateral root primordia (visualized in a longitudinal section in $\mathrm{J}$ ) and in meristematic cells of root tips (Figures S1A and S1B). In rhizobia-inoculated conditions, bacteria infection threads, revealed in magenta by the bacteria $\beta$-galactosidase activity, are indicated by arrows in (K)-(O). During early rhizobial infection events, MtBG2 promoter activity is limited to inner dividing root tissues (black arrowheads in K). When S. meliloti infection threads progress to the underlying cortex, GUS staining is progressively detected in nodule primordia tissues ( $\mathrm{L}-\mathrm{O}$ ) from dividing pericycle (a double arrowhead in the longitudinal section shown in $\mathrm{M}$ ) to middle or outer cortical and epidermal tissues (arrowheads in M-O). Note in (L)-(O) that the expression of MtBG2 (highlighted by white arrowheads) also accompanies the entry of rhizobial infection threads. $(\mathrm{J})-(\mathrm{O})$ are representative images from three independent experiments $(n=72)$. Because rhizobia infection is not synchronous, nodule primordia colonization events are not always observed in the same time period. Images in (J) and (K), (O), and (L)- $(\mathrm{N})$ were, respectively, taken at 3, 4, and 8 dpi with rhizobia. Boxes in (G) and (I) represent first and third quartile (horizontal box sides), minimum and maximum (outside whiskers). Central lines refer to median, and black circles depict mean values. Scale bars represent $50 \mu \mathrm{m}$. See also Figures S1 and S2. 
Quantification of the fluorescence signal in the pericycle, endodermis, and inner cortex of the spot-inoculated root regions confirmed the global downregulation of callose levels $1 \mathrm{dpi}$ (Figure $1 \mathrm{G}$ ). These results reveal that rhizobia promote a reduction in callose levels in inner root tissues within the first $24 \mathrm{hr}$ postinoculation and in a timescale that coincides with the establishment of symplastic connectivity between phloem and cortical tissues [34].

Regulation of symplastic transport during lateral root organogenesis in Arabidopsis thaliana is associated with the expression of callose-degrading PD-located $\beta$-1,3-glucanases [36, 38]. In order to identify PD-associated genes involved in root nodulation, we searched for putative $\beta$-1,3-glucanase-encoded proteins in $M$. truncatula, comprising the characteristic $\beta-1,3$-glucosidase enzymatic domain (also named glycosyl hydrolase like 17 [GHL17]) and other conserved features of PD-located Arabidopsis $\beta-1,3-$ glucanases, such as the presence of a secretory signal peptide (SP) and a glycosyl phosphatidylinositol anchor (GPI) (Table S1). Evolutionary relationships between the $M$. truncatula candidates with known PD proteins from Arabidopsis were established using phylogenetic analysis [39] (Figures $1 \mathrm{H}$ and $\mathrm{S} 1 \mathrm{~A})$. The tree topology was well supported by three complementary methods (Bayesian, maximum likelihood, and neighboring-joining algorithms) and highlighted Medtr8g085720 (MtBG1), Medtr3g083580 (MtBG2), Medtr4g132280 (MtBG3), Medtr3g065460 (MtBG5), and Medtr1g031900 (MtBG40) as GPI-anchored proteins most closely related to the Arabidopsis PD genes PdBG1 (AT3G13560), PdBG2 (AT2G01630), and PdBG3 (AT1G66250; Figures $1 \mathrm{H}$ and S1A) [38]. To determine which of these genes are regulated during the establishment of the $\mathrm{N}$-fixing symbiosis, we searched for those expressed in available rhizobial root hair and/or laser-dissected nodule zones (STAR Methods). qRT-PCR analyses then confirmed the upregulation of Medtr3g083580 (MtBG2) and Medtr3g065460 (MtBG5) gene expression in $M$. truncatula roots after inoculation with rhizobia (Figures $1 \mathrm{I}$ and S1C), in line with their expression levels in both infectome and nodule transcriptome databases. MtBG2 and MtBG5 are predicted to encode proteins with 498 and 491 amino acids, respectively, and comprise, in addition to an N-terminal signal peptide and an omega signal for GPI anchoring, a GHL17 and a carbohydrate-binding domain (X8) with similarities to PD-localized proteins (Figure S1B).

To summarize, $\beta$-1,3-glucanase-encoding genes are upregulated by rhizobia in a similar timescale as callose degradation, suggesting that these rhizobia-induced responses are interconnected.

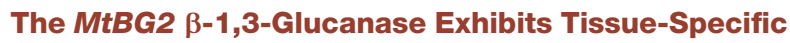
Symbiotic Expression Profiles in $M$. truncatula

To investigate whether the expression of MtBG2 coincides with sites of decreased callose levels (Figures 1A-1G), MtBG2 upstream sequences $(1.7 \mathrm{~kb})$ were used to drive the expression of the $\beta$-glucuronidase (GUS) reporter in transgenic $M$. truncatula roots generated via Agrobacterium rhizogenes transformation. In non-inoculated tissues, the expression of MtBG2 was restricted to the root tip and to developing lateral roots (Figures $1 \mathrm{~J}$ and $\mathrm{S} 2 \mathrm{~A}-\mathrm{S} 2 \mathrm{H}$ ). Upon rhizobial inoculation, the expression of MtBG2 was upregulated and associated with root nodule development (Figures 1K-1O and S2I-S2L). At early stages of infection, when rhizobia-containing infection threads have just initiated in root hairs or have reached at most the outer cortex, $p M t B G 2-d r i v e n$ expression can be visualized in dividing inner root cortical cell layers (Figures S2I and S2J). Sections validated GUS staining restricted to dividing inner tissues localized below an infection site (Figure 1K). At a later stage of nodule primordia development, when infection threads have already reached the second root cortical cell layer, GUS staining was more intense and broadly distributed from inner to outer root tissues (Figures 1M-10). GUS staining was not only detected in dividing inner tissues but was also found in middleto-outer cortical cells (and eventually the epidermis) directly associated with rhizobial infection thread progression (Figures 1L-10). Longitudinal root sectioning confirmed the expression of MtBG2 in dividing inner tissues (from pericycle to inner cortex) and in nondividing infection thread-containing cortical cells (Figure 1M). In mature nodules, GUS staining was restricted to the apical zone, which includes both meristematic and early infection zones (Figures S2K and S2L).

Taken together, our results indicate that rhizobial inoculation induces the expression of MtBG2 in dividing inner pericycle and cortical tissues, where callose levels are coincidently reduced, and subsequently in outer tissues preparing or undergoing rhizobial infection.

Rhizobial Inoculation Promotes Cell-to-Cell Symplastic Connectivity in Outer Root Tissues Undergoing Infection The initial expression of MtBG2 in dividing cells supports an active role in the creation of symplastic connections in nodule primordia initials, in line with previous findings [34]. However, the progressive outward expression of MtBG2 also favors the hypothesis that symplastic connections are established in nondividing outer root cells undergoing rhizobial infection (Figures $1 \mathrm{~L}-10$ ). To explore changes in epidermal-cortical symplastic connectivity during rhizobial infection, we traced the transport of the carboxy-fluorescein diacetate (CFDA) symplastic tracer (STAR Methods) in roots after rhizobial inoculation. CFDA is a non-fluorescent and membrane-permeable dye that once inside the cell is cleaved by endogenous esterases to produce a permeable fluorescent form that, within a given period, can only move between cells via intercellular PD connections. The diffusion of the CFDA-derived fluorescent molecule was followed from outer to inner root tissue layers in both mock and rhizobia-inoculated conditions. The green fluorescence signal was restricted to epidermal tissues of mock control roots (Figure $2 \mathrm{~A}$ ) but clearly diffused from epidermal to underlying cortical cells at $3 \mathrm{dpi}$ in rhizobia-inoculated tissues (Figures $2 \mathrm{~B}$ and $2 \mathrm{C}$ ). To verify this, mobile YFP (yellow fluorescent protein) that can freely diffuse through $\mathrm{PD}$ and the non-mobile ER-tagged mCherry-HDEL protein were co-expressed in the root epidermis of $M$. truncatula roots, under the epidermal-specific EXPA promoter [30]. As expected, the red mCherry-HDEL fluorescence was restricted to the root epidermis in both inoculated and non-inoculated conditions (Figures 2D, 2E, 2G, and 2H), whereas the green YFP signal diffused, presumably via PD, from the root epidermis to the underlying cortex only in rhizobia-inoculated conditions (compare rhizobia-inoculated roots in Figures $2 \mathrm{E}$, $2 \mathrm{~F}$, and $2 \mathrm{H}$ with mock control in $2 \mathrm{D}$ ).

Together, these results indicate that epidermal and cortical cells become symplastically connected during early stages of rhizobial root colonization. 

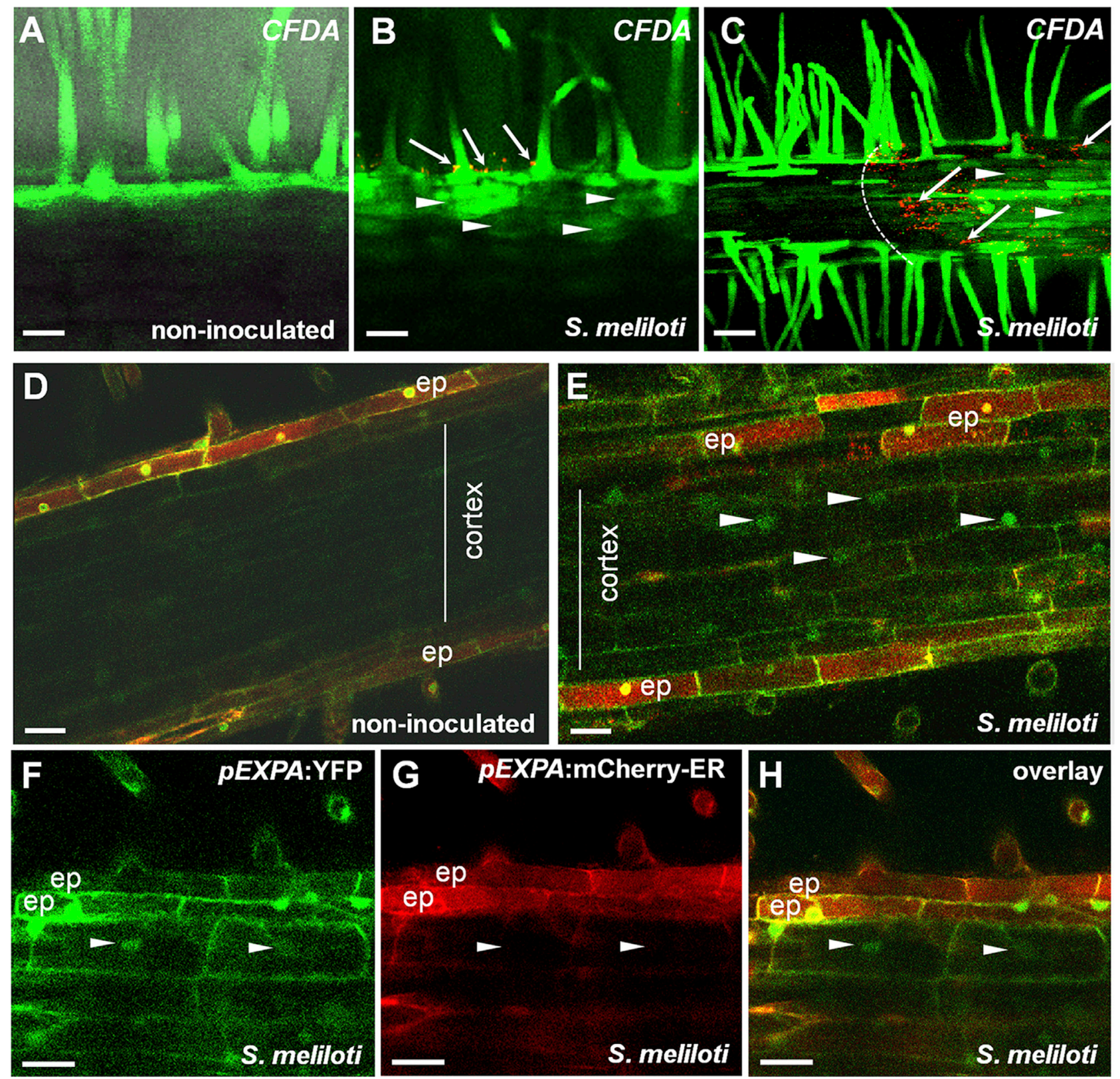

Figure 2. Rhizobia-Induced Changes in Root Intercellular Symplastic Connectivity

(A-C) M. truncatula roots were spot inoculated with a $S$. meliloti mCherry strain and, at 3 dpi, exposed to the symplastic tracer CFDA. In control non-inoculated roots (A), the green CFDA-derived fluorescence is restricted to the root epidermis and root hairs. In bacterial-spot-inoculated roots (B and C; 3 dpi), the green CFDA-derived fluorescence is clearly visible in cortical cells (arrowheads). Arrows in (B) and (C) highlight red fluorescent bacteria. Note that green fluorescence in the cortex is preferentially visualized at the spot-inoculated root zone (at the right side of the dotted line in C).

(D-H) M. truncatula roots co-expressing a mobile YFP (green or yellow fluorescence seen in both cytoplasm and nuclear compartments) and an endoplasmicreticulum-tagged mCherry-ER (red fluorescence) under the control of the epidermis-specific promoter pEXPA were inoculated (E-H) or not (D) with a S. meliloti CFP strain and analyzed $3 \mathrm{dpi}$. The green or yellow YFP and red mCherry-ER fluorescence is restricted to the root epidermis (ep) in non-inoculated roots in (D). Upon inoculation, the green or yellow fluorescence is detected in cells in the root epidermis (ep in E, $F$, and $H$ ) and in the root cortex (white arrowheads in $E, F$, and $H$ ), and the red $m$ Cherry-ER fluorescence remains restricted to the root epidermis (ep in E, G, and $\mathrm{H}$ ) and is not transported to underlying cortical cells (white arrowheads in $\mathrm{G}$ ). Scale bars represent $100 \mu \mathrm{m}(C), 50 \mu \mathrm{m}(\mathrm{A}$ and $\mathrm{B})$, and $20 \mu \mathrm{m}(\mathrm{D}-\mathrm{H})$.

MtBG2 Is a PD-Localized Callose-Degrading $\beta-1,3-$ Glucanase that Regulates Symplastic Flux and Root Nodule Development in M. truncatula

Symplastic communication is established in rhizobia-inoculated tissues [34] (Figure 2), where MtBG2 is shown to be progressively expressed (Figures $1 \mathrm{~K}-10$ ). In order to investigate whether $M t B G 2$ is actively involved in rhizobia-induced degradation of callose at PD sites, we first evaluated MtBG2 localization. To this end, a fluorescent protein fusion was generated for subcellular localization in $M$. truncatula roots produced via 

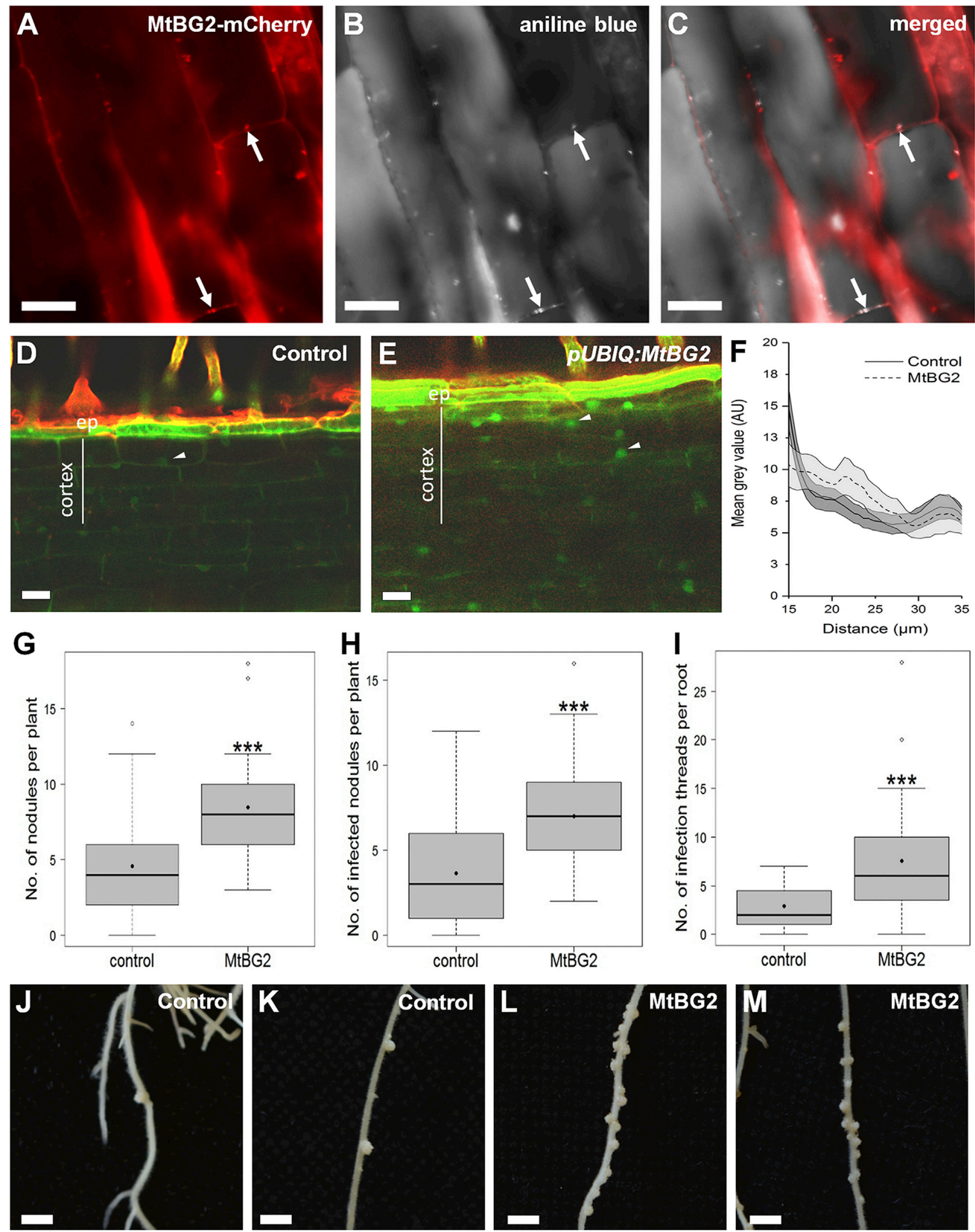

(legend on next page) 
A. rhizogenes transformation. The fluorescent fusion was created by inserting the mCherry coding sequence between MtBG2 amino acids 462 and 463, just before the predicted omega site, and was expressed in M. truncatula under the constitutive Arabidopsis UBIQ promoter. As shown in Figures $3 \mathrm{~A}-3 \mathrm{C}$, the protein localized to the cell periphery at callose enriched PD sites, which are revealed using aniline blue staining. To determine whether MtBG2 encodes an active enzyme, we investigated callose levels in $M$. truncatula roots constitutively expressing the MtBG2mCherry fusion protein. Immunolocalization in root sections and aniline blue staining revealed callose-associated fluorescence signals strongly reduced in MtBG2-overexpressing roots compared to control roots transformed with an empty vector (Figures S2M-S2P). Integration analysis of aniline blue fluorescence signal in multiple sections confirmed a significant decrease in callose in MtBG2-overexpressing roots consistent with a role in callose degradation (Figure S2Q).

In order to investigate whether degradation of callose by MtBG2 affects symplastic communication, we monitored the symplastic transport of a mobile GFP version in roots ectopically expressing MtBG2. A stable $M$. truncatula line expressing GFP under the epidermis-specific promoter $p E X P A$ was transformed to co-express a control empty vector (Figure 3D) or a $p U B I Q: M t B G 2$ construct (Figure 3E). The green GFP signal is detected in the root epidermis and diffuses to the underlying cortical cells in rhizobia-inoculated conditions in both control and pUBIQ:MtBG2 roots (compare Figures $3 D$ and $3 E$ ). However, GFP diffusion is significantly increased in $p U B I Q: M t B G 2$ roots, as reported by quantification of the signal in root cortical cells (Figure 3F). The data suggest that MtBG2 expression positively affects symplastic communication.

Phenotypic analysis of transgenic roots expressing $p U B I Q-$ MtBG2 was carried out in parallel. MtBG2-expressing roots were not substantially affected in their development but exhibited a significant increase in nodule number compared to control roots at $7 \mathrm{dpi}$ with rhizobia (Figures $3 \mathrm{G}$ and $3 \mathrm{~J}-3 \mathrm{M}$ ). These developed nodules were efficiently colonized by the symbiotic bacteria, as scored by the number of nodules containing histochemically stained lacZ-expressing rhizobia (Figure $3 \mathrm{H}$ ). Interestingly, the number of root hair infection threads was also significantly increased in pUBIQ-MtBG2 roots (Figure 3I), sug- gesting that ectopic expression of MtBG2 positively influences both infection and nodule organogenesis.

In summary, MtBG2 constitutes a new callose-degrading, PDassociated enzyme that regulates intercellular symplastic transport and root nodule development and infection in $M$. truncatula.

\section{Establishment of Symplastic Connectivity Is Important for Root Endosymbiotic Colonization}

Because ectopic expression of MtBG2 induces an increase in cell-to-cell connectivity, we decided to evaluate whether obstruction of root symplastic fluxes can affect root nodulation. For this, we made use of a mutant version of the callose synthase gene CALS3 (cals3m), previously shown to occlude PD in Arabidopsis by the localized overproduction of callose in cell walls [37]. Expression of cals $3 m$ under the UBIQ promoter in Nicotiana benthamiana leaves confirmed the ability of cals $3 m$ to overproduce PD-localized callose by comparison with the mock control (Figures S3F and S3G). To evaluate the effect of PD obstruction in rhizobium-inoculated $M$. truncatula roots, we expressed cals $3 m$ under the control of rhizobia-induced symbiotic promoters: pMtBG2 (Figures 1K-1O and S2A-S2L); $p E R N 1$ [18]; or pANN1 [40], and the epidermal-specific promoter pEXPA. These promoters display partially overlapping expression patterns during root nodule development (Figure S3A). Although pMtBG2-driven symbiotic expression initiates in inner tissues and propagates toward infected tissues, pERN1- and pANN1driven expression initiates in outer root tissues (Figure S3A). In rhizobia-inoculated roots, $p E R N 1$-driven expression is detected very early (within hours) in the root epidermis of the symbiotic responsive zone before being associated with cells preparing and undergoing infection (from 3 dpi onward) in epidermal and outer cortical tissues and then in nodule primordia cells before and during infection [18]. pANN1-driven expression is higher in outer root tissues, but it is also associated with the root endodermis and epidermis of infection sites and detected later in developing nodules [40]. As illustrated for rhizobia-inoculated $p E R N 1$-cals3m, ectopic expression of cals $3 m$ increases callose levels in $M$. truncatula roots (Figures S3H-S3K).

Transgenic roots expressing the pMtBG2:cals3m construct showed a partial but significant reduction in the number of nodules and infected nodules formed at $7 \mathrm{dpi}$ with rhizobia, compared to control roots expressing pMtBG2:GFP (Figures

Figure 3. The $\beta-1,3-G l u c a n a s e ~ M t B G 2$ Localizes at Plasmodesmata and Regulates Symplastic Flux and Nodulation in M. truncatula $(A-C)$ MtBG2 fused to mCherry was expressed under the ubiquitin promoter ( $p U B / Q)$ in transgenic M. truncatula roots. MtBG2-mCherry localizes in the cell periphery and accumulates in putative PD sites (white arrows in A). Callose deposition at PD is revealed in (B) by aniline blue staining (false colored white). Merged images shown in (C) highlight the co-localization of MtBG2-mCherry with callose deposits at PD (white arrows).

(D-F) A stable $M$. truncatula line expressing a mobile GFP version under the control of the epidermis-specific promoter $p E X P A$ was used to generate transgenic roots co-expressing a control empty vector (D) or pUBIQ:MtBG2 (E). Roots were imaged 5 dpi with rhizobia using confocal microscopy. Counterstaining with propidium iodide reveals the epidermal cell layer in red. GFP fluorescence (green) is expressed in the root epidermis (ep in D and E), but it is also detected in cortical tissues presumably due to symplastic diffusion (arrowheads). (F) shows quantification of the GFP signal (using ImageJ mean gray values as a.u.) in cortical cell layers ( $x$ axis represents distance from the epidermis) in three independent biological replicas and in a region of interest $55 \mu m$ wide $(n>5)$. The gray area above and below the mean (continuous line for control; discontinuous line for MtBG2) shows SEs. Notice that mean values in MtBG2 are above control, indicating increase in GFP diffusion.

(G-M) M. truncatula roots constitutively expressing MtBG2 showed significantly higher number of nodules (at 7 dpi in G and at 15 dpi as illustrated in J-M), rhizobia-colonized nodules and nodule primordia $(\mathrm{H})$, and root hair infection threads $(\mathrm{l})$ at 7 dpi compared to control roots expressing an empty vector $(n=26$ in $G$ and $\mathrm{H}$ and $\mathrm{n}=27$ in I independent samples). A Mann-Whitney test (G-I) was performed in $\mathrm{R}$ (asterisks indicate statistical difference compared with vector control; ${ }^{* \star *} \mathrm{p}<0.001$ ). Boxplots represent first and third quartile (horizontal box sides), minimum and maximum (outside whiskers), median (central lines), and mean (black circles) values.

Scale bars represent $20 \mu \mathrm{m}$ in (A)-(E) and $1 \mathrm{~cm}$ in $(J)-(M)$. See also Figure S2. 
A

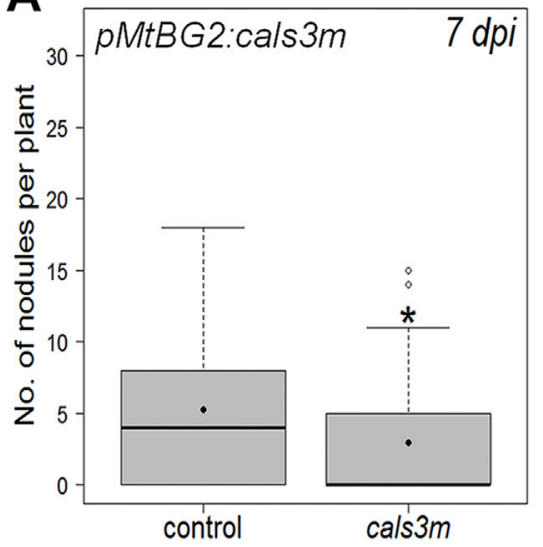

B

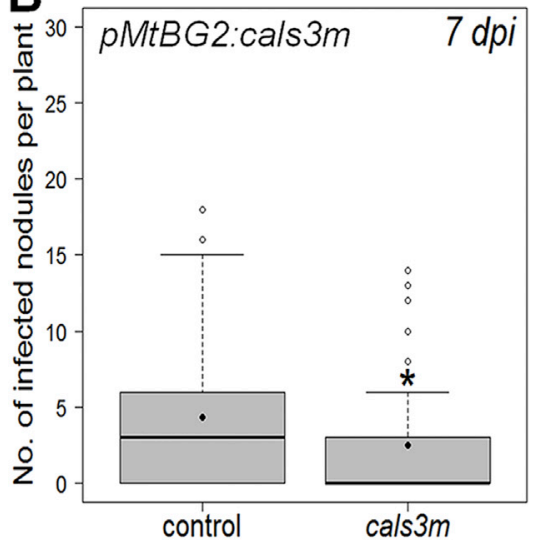

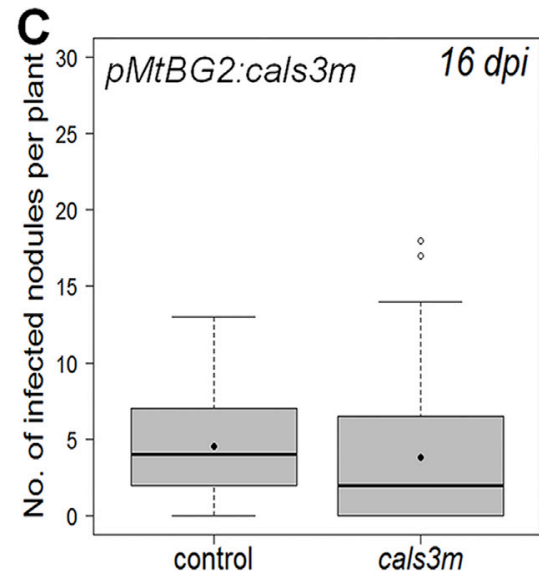
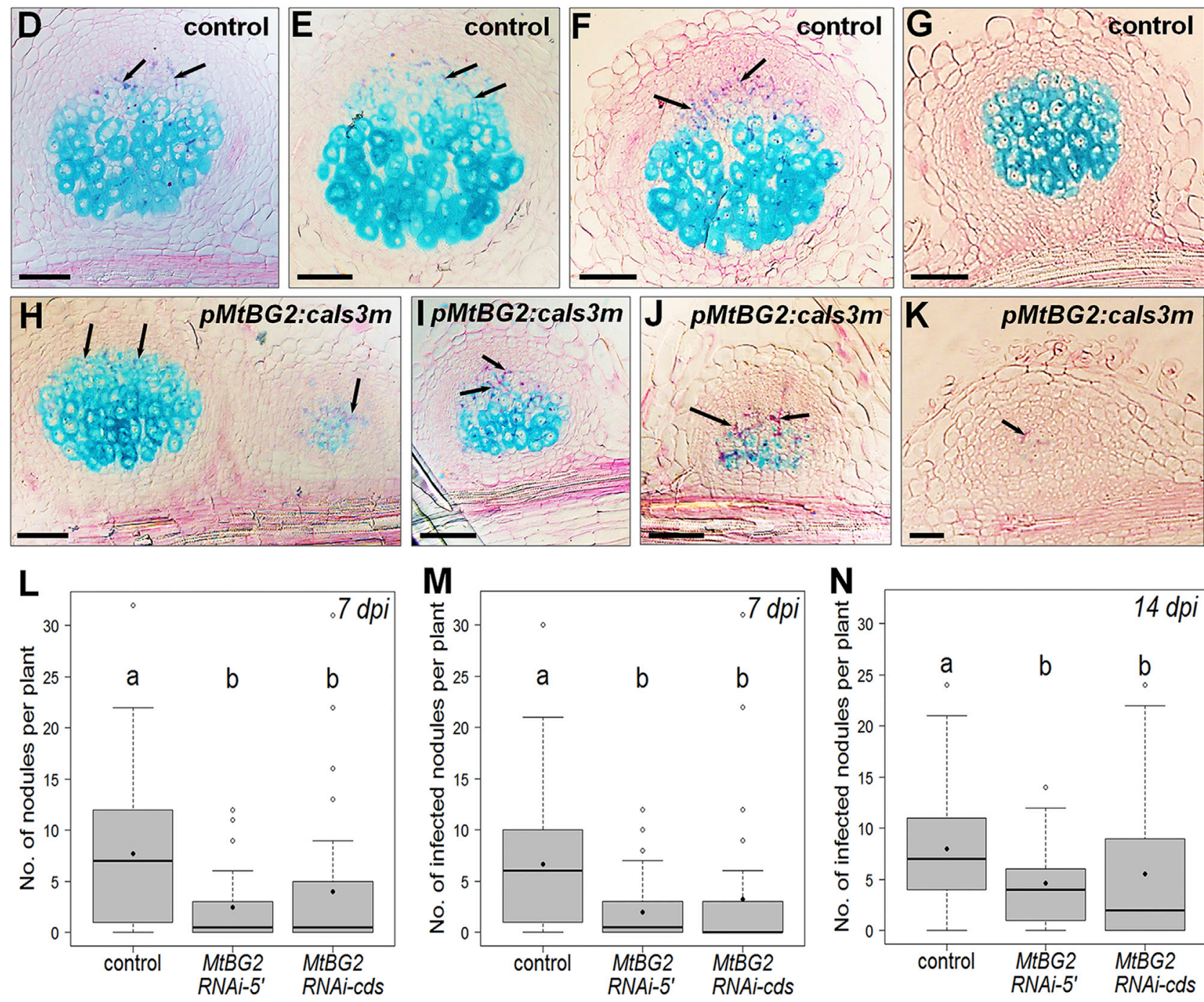

Figure 4. Ectopic Expression of the Callose Synthetic Enzyme cals3m under the MtBG2 Promoter or RNAi Silencing of the Endogenous MtBG2 Gene Affects Nodule Development and Colonization

(A-C) M. truncatula roots expressing the callose synthase synthetic mutation cals $3 m$ under the control of $M$ tBG2 promoter sequences were inoculated with a S. meliloti lac $Z$ strain and analyzed 7 and $16 \mathrm{dpi}$. Total number of nodules $(A)$ and rhizobia-colonized nodules and nodule primordia (B and $C)$ were scored per root system at $7 \mathrm{dpi}$ ( $A$ and $B$ ) and $16 \mathrm{dpi}(C)$. Boxplots in (A)-(C) represent values from control ( $n=45$ for $7 \mathrm{dpi} ; n=57$ for $16 \mathrm{dpi})$ and cals $3 m$ ( $n=41$ for $7 \mathrm{dpi} ; n=52$ for $16 \mathrm{dpi}$ ) plants from three independent experiments. A Mann-Whitney test was performed in $\mathrm{R}$ (asterisks indicate statistical difference compared with GFP control; $\left.{ }^{*} \mathrm{p}<0.05\right)$. 
$4 \mathrm{~A}$ and $4 \mathrm{~B}$ ). $69 \%$ of pMtBG2:GFP control roots exhibited colonized nodules/primordia (an average number of 4.3 per root system), and pMtBG2:cals3m exhibited a lower frequency (49\%) of colonized nodules/primordia (an average of 2.5 per root system). Although the reduction in the number of colonized nodules is also observed at $16 \mathrm{dpi}$ (Figure 4C), the difference is not supported by statistic tests, likely due to the overall variability among individual plants. However, a close microscopic analysis revealed that nodules in pMtBG2:cals3m roots are less differentiated and colonized (Figures $4 \mathrm{H}-4 \mathrm{~K}$ ) compared to the pMtBG2:GFP control (Figures 4D-4G).

A more drastic phenotype was observed in transgenic roots expressing the pERN1:cals3m construct, which displayed a highly significant reduction in the number of nodules formed at $7 \mathrm{dpi}$ compared to the $p E R N 1$ :GFP control (Figure 5A). Although the majority (79\%) of $p E R N 1$ :GFP control roots exhibited colonized nodules/primordia (an average number of 6 per root system), only a small proportion (28\%) of $p E R N 1: c a l s 3 m$ roots exhibited colonized nodules/primordia and at a low frequency (an average of 0.8 per root system; Figure 5B). This low frequency was also observed at $16 \mathrm{dpi}$ (Figure 5C), indicating that the colonization defect is maintained over time. A close view of rhizobia-inoculated roots revealed that the few nodules formed in pERN1:cals $3 m$ roots were under-differentiated and exhibited mostly growing infection threads (Figures $5 \mathrm{G}$ and $5 \mathrm{H}$ ), compared to control roots at $7 \mathrm{dpi}$ with rhizobia (Figures 5D and 5E). At a subsequent developmental stage, mature $p E R N 1: c a l s 3 m$ nodules exhibited abnormally long infection threads (Figure 5l) compared to control nodules (Figure 5F), suggesting a defect in bacterial release into plant cells [41]. Moreover, $p E R N 1$ : cals $3 m$ roots also showed non-colonized nodule primordia with arrested infection threads in outer epidermal and cortical tissues, reinforcing the defective infection phenotype upon cals $3 m$ expression. To verify that this strong phenotype correlates with changes in symplastic connectivity, we used a stable transgenic line expressing mobile free GFP under the EXPA promoter (Figures $5 \mathrm{~L}-5 \mathrm{~N}$ ). As before, rhizobia-inoculated control roots showed GFP expression in epidermal and cortical tissues (presumably due to symplastic diffusion). In contrast, expression was mostly restricted to the root epidermis in $p E R N 1: c a l s 3 m$ roots at $5 \mathrm{dpi}$ (Figures $5 \mathrm{M}$ and $5 \mathrm{~N}$ ). A nodulation-defective phenotype was also observed in roots expressing cals $3 m$ under the $p A N N 1$, but not under the epidermis-specific $p E X P A$ promoter (Figure S4), suggesting that regulation of callose in the root epidermis is not sufficient to restrict nodulation.

Because the expression of cals $3 m$ under the ERN1 promoter is sufficient to alter root nodulation, we also tested whether pERN1:MtBG2 RNAi constructs (targeting $5^{\prime}$ untranslated or coding sequences of $M t B G 2)$, aiming to downregulate MtBG2 in ERN1 expression domains, could also affect root nodulation. Following the same trend as pMtBG2:cals3m, MtBG2 RNAi constructs led to reduced root nodulation and colonization at 7 and $14 \mathrm{dpi}$ with rhizobia (Figures $4 \mathrm{~L}-4 \mathrm{~N}$ and S5B), although not strongly affecting general root weight (Figure S5A). We performed complementary experiments using a $p E R N 1$-driven PD-associated beta-1,3-glucanase ( $P d B G 1$ ) previously characterized in Arabidopsis [38]. PdBG1 remains PD associated in M. truncatula transgenic roots (Figures S5D-S5F), and its expression under $p E R N 1$ did not significantly affect root development (Figure S5C). Similarly to $p U B Q: M t B G 2$, these plants exhibited significant increase in the number of nodules and infected nodules at 16 dpi (Figures S5C and S5G-S5I).

Together, these findings indicate that callose regulation and symplastic communication in tissues overlapping ERN1/ANN1/ MtBG2 expression domains are important for nodule development and colonization.

\section{Symplastic Connectivity Is Required for the Transcriptional Activation of NIN and Its Targets in Developing Nodules}

Because obstruction of root symplastic communication led to strong root nodulation phenotypes, we monitored how the expression of the key symbiotic regulators ERN1 [12, 13] and NIN [10] and associated targets were affected in this context. Transcript levels of ERN1 and its target ENOD11 [13] were found to accumulate at a slightly higher (for ERN1) or comparable levels (for ENOD11) in cals3m (rhizobia-inoculated pERN1:cals3m) compared to control (rhizobia-inoculated $p E R N 1: G F P$ ) samples at $16 \mathrm{dpi}$ (Figures $6 \mathrm{~A}$ and $6 \mathrm{~B}$ ). In contrast, the expression of NIN and its target NF-YA1 [42] were strongly reduced in pERN1: cals $3 m$ by comparison with the $p E R N 1$ :GFP control (Figures $6 \mathrm{C}$ and 6D). To investigate at which symbiotic stage their downregulation occurs, we analyzed the expression of these and other symbiotic genes (NPL1 [21], CRE1 [43], CLE12, and CLE13 [32]) by qRT-PCR before (at $3 \mathrm{dpi}$ ) and after (at $7 \mathrm{dpi}$ ) developing nodules and nodule primordia were detected (via microscopy) in rhizobia-inoculated roots. At 3 dpi, ENOD11, ERN1, NIN, CLE12, CLE13, CRE1, and NPL1 were expressed at similar levels in mock or rhizobia-inoculated roots of both $p E R N 1$ :GFP control and $p E R N 1: c a l s 3 m$ roots (Figures $\mathrm{S} 6 \mathrm{~A}-\mathrm{S} 6 \mathrm{H}$ ), suggesting that callose overaccumulation does not strongly affect gene expression during early stages of root colonization. However, a clear reduction in the expression of NIN and associated targets NF-YA1/CLE12/CLE13 was observed in pERN1:cals3m roots at 7 dpi (Figures S6K-S6N and S6P), although the expression of other symbiotic genes was not strongly affected (Figures S6I,

(D-K) Illustrative images of developing nodules formed in $M$. truncatula roots expressing the control vector pMtBG2:GFP (D-G) or pMtBG2:cals3m (H-K) 7 dpi with rhizobia. Pictures show longitudinal sections counterstained with Ruthenium Red and X-Gal, which, respectively, reveal cell outlines in magenta and, in magenta or blue, the bacteria $\beta$-galactosidase activity in infection threads (arrows) and differentiating bacteroid rhizobia. Note in $(\mathrm{H})-(\mathrm{K})$ that nodules formed in pMtBG2:cals3m roots are overall less differentiated or colonized than those of the control GFP in (D)-(G).

$(\mathrm{L}-\mathrm{N})$ MtBG2 RNAi-5' and MtBG2 RNAi-cds constructs targeting the $5^{\prime}$ UTR or coding sequence (cds) of MtBG2 were expressed under the control of the ERN1 promoter in $M$. truncatula transgenic roots that were inoculated with a S. meliloti lacZ strain and analyzed $7 \mathrm{dpi}$ and $14-16 \mathrm{dpi}$. Number of nodules (L) and rhizobiacolonized nodules and nodule primordia ( $\mathrm{M}$ and $\mathrm{N}$ ) were scored per root system at $7 \mathrm{dpi}(\mathrm{L}$ and $\mathrm{M})$ and $14-16 \mathrm{dpi}(\mathrm{N})$ and compared to transgenic $p E R N 1: G U S$ reporter transformed roots (control). Boxplots represent values from control GUS ( $\mathrm{n}=63 \mathrm{for} 7 \mathrm{dpi} ; \mathrm{n}=58$ for $14-16 \mathrm{dpi}$ ), MtBG2 RNAi-5' $\mathrm{n}=38$ for $7 \mathrm{dpi}$; $\mathrm{n}=31$ for 14-16 dpi), and MtBG2 RNAi-cds ( $n=34$ for $7 \mathrm{dpi} ; n=35$ for $14-16 \mathrm{dpi}$ ) from three independent experiments. Kruskal-Wallis test of the values were performed in $R$. Classes sharing the same letter are not significantly different $(p<0.001$ in $L$ and $M ; p<0.01$ in $N)$.

Scale bars in (D)-(K) represent $100 \mu \mathrm{m}$. See also Figures S3 and S5. 

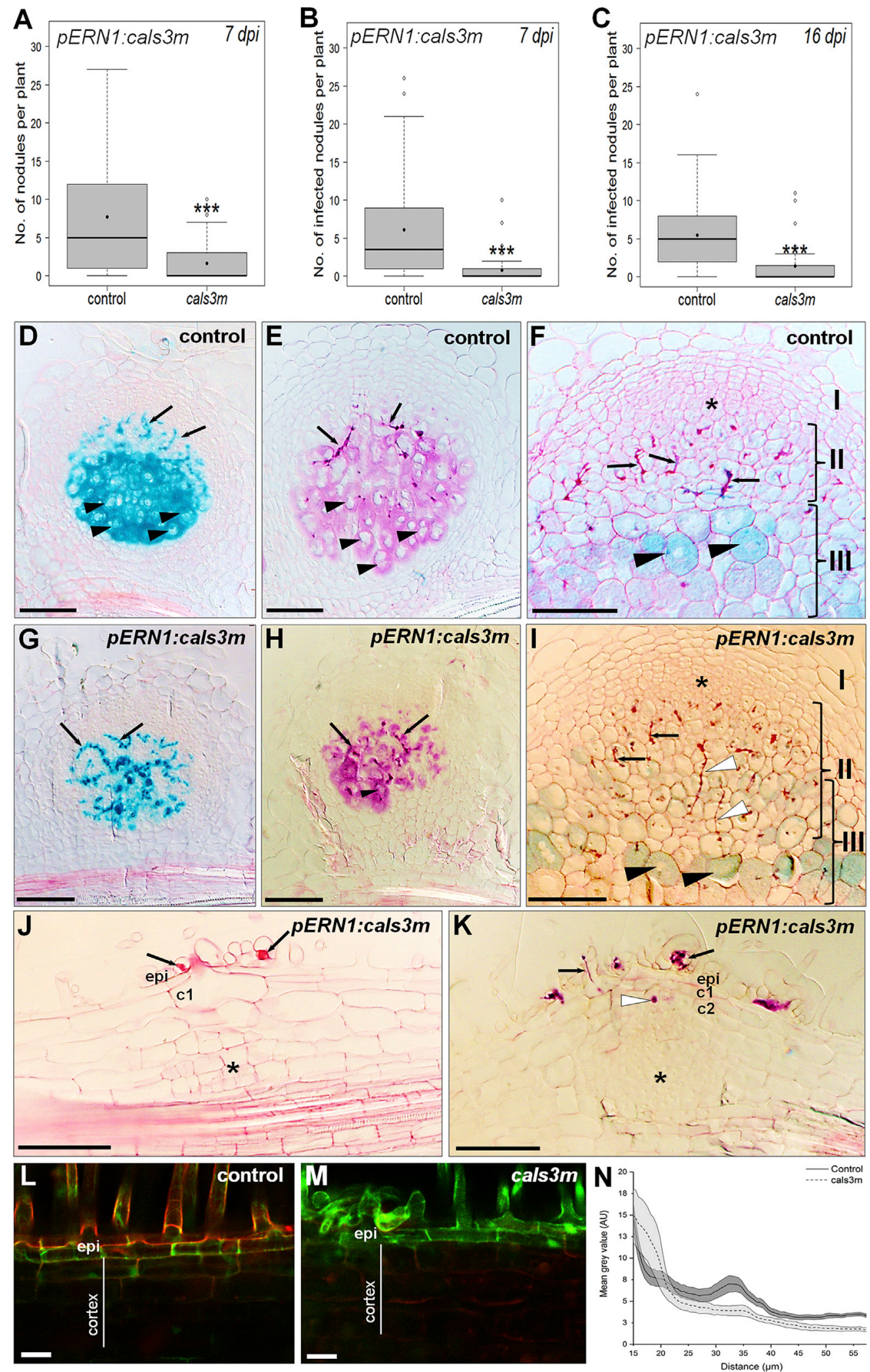

(legend on next page) 
S6J, and S6O). These results suggest that the selective downregulation of NIN and its targets is associated with the development of nodule primordia or nodule structures. To verify this hypothesis, the tissue-specific promoter activity of NIN was investigated in control and $p E R N 1: c a l s 3 m$ roots using $p N I N-G U S$ fusions. Although not functionally validated before, the $2-\mathrm{kb}$ and $1.4-\mathrm{kb}$ NIN promoter sequences used here were sufficient to confer NIN expression during infection and nodule organogenesis (Figures 6 and S7). A basal level of NIN expression was observed in the root apex, lateral root primordia or epidermal and outer cortical tissues of non-inoculated roots (Figures S7A-S7C). At the root infection zone, GUS activity was clearly more intense in the root epidermis and outer cortex at localized rhizobial infection sites (Figures 6K and S7D-S7F, S7H, and S7J). In developing nodule primordia, GUS activity was associated with both infection sites and underlying dividing cells in the middle and inner cortex (Figures 6E-6G, 6L, and 6M). In transgenic roots co-expressing the pERN1:cals3m construct, GUS activity was observed at infection sites (Figures 6H-6J, 6N-6P, S7G, S7I, and S7K); however, GUS staining in the middle or inner cortex of nodule primordia formed in pERN1:cals3m (Figures 6H-6J and 6N-6P) was not evident when compared to control roots (Figures 6E$6 \mathrm{G}$ and $6 \mathrm{~K}-6 \mathrm{M})$. Similar results were obtained when analyzing the expression of a pNF-YA1:GUS fusion [42] co-transformed with pERN1:cals3m (Figures S7M-S7O) compared to control roots (Figure S7L). Taken together, our findings suggest that the expression of NIN (and downstream NF-YA1) in the middle and/or inner cortex of developing nodules is dependent on the establishment of symplastic connectivity and, potentially, on yet uncharacterized symplastically regulated signals.

\section{DISCUSSION}

In this manuscript, we provide evidence that symplastic communication is necessary for endosymbiotic colonization during legume root nodulation. We report new findings on the importance of the regulation of callose in inner root tissues for the initiation and development of nodule primordia. Callose levels decrease in inner pericycle and cortical root tissues as early as 1 day after spot inoculation with rhizobia (Figure 1), which overlaps with the onset of the first set of cell divisions (18-24 hr) [3]. Reduction in callose levels coincides with the localized endogenous expression of a novel PD-localized $\beta-1,3-$ glucanase from $M$. truncatula (MtBG2) that degrades callose and regulates symplastic communication at PD sites (Figures 3 and S2). This supports an active role for MtBG2 in creating symplastic domains to connect nodule primordia initials, in agreement with previous work describing the formation of phloem-to-inner cortex symplastic continuity associated with initial cell divisions [34]. Similar remodeling in symplastic connectivity was reported to control lateral root initiation and patterning, regulated by the closely related $\beta-1,3$-glucanases PdBG1 and PdBG2 in Arabidopsis [38]. It is thus conceivable that MtBG2, also expressed in lateral root primordia (Figures 1 and S2), was recruited from a basal root developmental function to regulate the formation of nodule primordia in legumes. Phenotypic and gene expression analyses of transgenic plants affected in callose metabolism clearly indicate that PD regulation is an important early step for the successful establishment of the rhizobia-legume association, as illustrated in a model presented in Figure 7.

In addition to its early expression in dividing nodule initials, MtBG2 expression progressively extends outward to the outer cortex and epidermal cell layers, where new symplastic connections are created, as shown by the transport of fluorescent molecules in vivo (Figures 2 and 3). These findings indicate that symplastic communication is not only established between dividing cells but also in root cells actively preparing for endosymbiotic accommodation. We have evaluated the importance of this symplastic connectivity by the tissue-specific expression of a hyperactive callose synthase (cals $3 m$ ) shown before to enhance

Figure 5. M. truncatula Roots Expressing cals3m under the Control of the Infection-Related ERN1 Promoter Are Defective in Symbiotic Colonization and Symplastic Communication

(A-C) M. truncatula roots expressing the callose synthase mutant version cals $3 m$ under the control of the infection-related promoter ERN1 were inoculated with a S. meliloti lac $Z$ strain and analyzed $7 \mathrm{dpi}$ and $16 \mathrm{dpi}$. Total number of nodules (A) and rhizobia-colonized nodules and nodule primordia (B and C) were scored per root system at $7 \mathrm{dpi}(\mathrm{A}$ and $\mathrm{B}$ ) and $16 \mathrm{dpi}(\mathrm{C})$. Boxplots in (A)-(C) represent values from control roots expressing $p E R N 1$ :GFP $(\mathrm{n}=66$ for $7 \mathrm{dpi} ; \mathrm{n}=41$ for $16 \mathrm{dpi})$ or $p E R N 1$ :cals $3 m$ ( $\mathrm{n}=79$ for $7 \mathrm{dpi} ; \mathrm{n}=43$ for $16 \mathrm{dpi}$ ) obtained from five ( $7 \mathrm{dpi}$ ) and two (16 dpi) independent experiments. First and third quartile (horizontal box sides), minimum and maximum (outside whiskers), median (central lines), and mean (black circles) values are depicted. A Mann-Whitney test was performed in $\mathrm{R}$ (asterisks indicate statistical difference compared with control; ${ }^{* \star} p<0.001$ ).

(D-K) Representative images of $M$. truncatula nodules from control $p E R N 1$ :GFP transformed roots (D and F), non-transformed roots (E), or $p E R N 1$ :cals3m (G-K) composite plants taken $7 \mathrm{dpi}(\mathrm{D}, \mathrm{E}, \mathrm{G}, \mathrm{H}$, and $\mathrm{J}$ ) or $12 \mathrm{dpi}(\mathrm{F}, \mathrm{I}$, and $\mathrm{K})$. Pictures represent longitudinal sections counterstained with Ruthenium Red (cell outlines in magenta) and X-Gal or Mag-Gal, which label in blue or magenta the rhizobial infection threads (arrows) and the differentiated rhizobia. Young developing nodules in control roots contain growing infection threads (arrows in D-F) and recently released rhizobia differentiating into the nitrogen-fixing bacteroid form (in blue or magenta, as indicated by arrowheads in $\mathrm{D}$ and $\mathrm{E}$ ). At a subsequent developmental stage, nodules are recognized by the presence of an apical meristematic zone (asterisks, zone I) followed by a clearly defined infection-thread-containing invasion zone II and a zone III with differentiated cells containing rhizobia at the cell periphery (arrowheads, F). The few infected nodules formed in cals $3 m$ roots are under-differentiated and contain essentially growing infection threads (arrows) and only a few differentiated rhizobia (arrowheads) at $7 \mathrm{dpi}(\mathrm{G}$ and $\mathrm{H})$ compared to control nodules ( $\mathrm{D}$ and $\mathrm{E})$. Nodule zonation of mature cals $3 \mathrm{~m}$ nodules (I) is less clearly defined and comprise abnormally long infection threads (white arrowheads), which is reminiscent of defective bacterial release into plant cells.

( $\mathrm{J}$ and K) At 7 and $12 \mathrm{dpi}$, cals $3 \mathrm{~m}$ roots exhibited non-colonized nodule primordia, which were not observed in control roots. Infection threads (arrows) are visualized in outer epidermal (epi) and/or outer cortical tissues but do not reach the dividing nodule primordia tissues in the cortex (asterisks).

(L-M) A stable pEXPA:GFP transgenic line was transformed to co-express a control empty vector (L) or pERN1:cals3m (M). Representative confocal images (control counterstained in red with propidium iodide) of the transgenic roots are shown 5 dpi with rhizobia. GFP fluorescence (green) appears restricted to the epidermal cell layer in the cals $3 m$, suggesting reduced symplastic connectivity.

(N) Quantification of the GFP signal (using ImageJ mean gray values as a.u.) in cortical cell layers ( $x$ axis represents distance from the epidermis) in three independent biological replicas and in a region of interest $55 \mu \mathrm{m}$ wide $(n>5)$. The gray area above and below the mean shows SEs.

c1 and c2, cortical cell layers 1 or 2; epi, epidermis. Scale bars represent $100 \mu \mathrm{m}$ in (D)-(K) and $20 \mu \mathrm{m}$ in (L) and (M). See also Figures S3 and S4. 

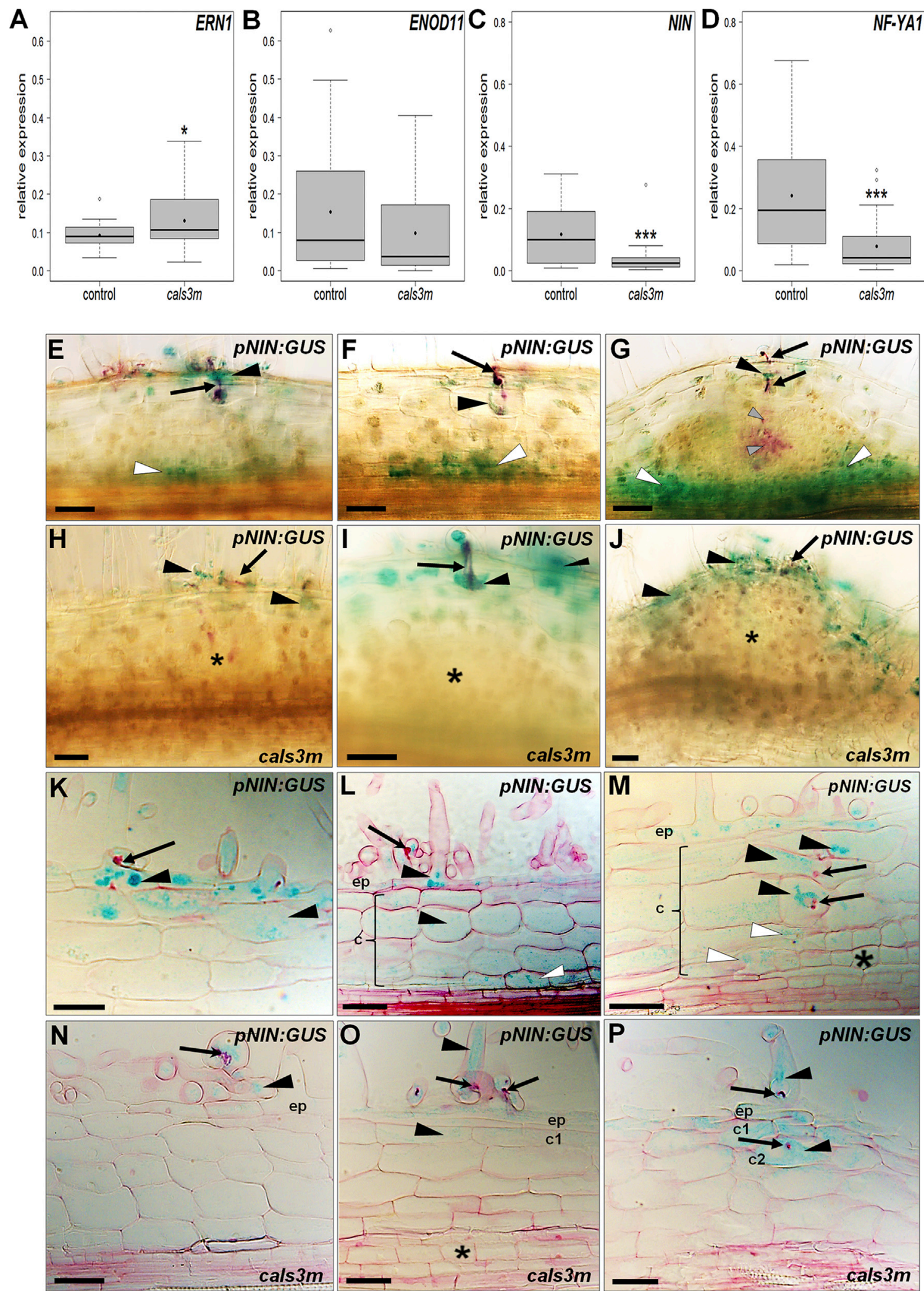

Figure 6. Symplastic Connectivity Is Important for the Expression of NIN in the Cortex

(A-D) qRT-PCR analyses of ERN1 (A), ENOD11 (B), NIN (C), and NF-YA1 (D) transcripts in total RNA samples of nodulated roots (16 dpi) from individual M. truncatula composite plants expressing cals $3 m(\mathrm{cal} / \mathrm{s} 3 \mathrm{~m})$ or the GFP control (control), under the $p E R N 1$. qRT-PCR analyses of these and other symbiotic 


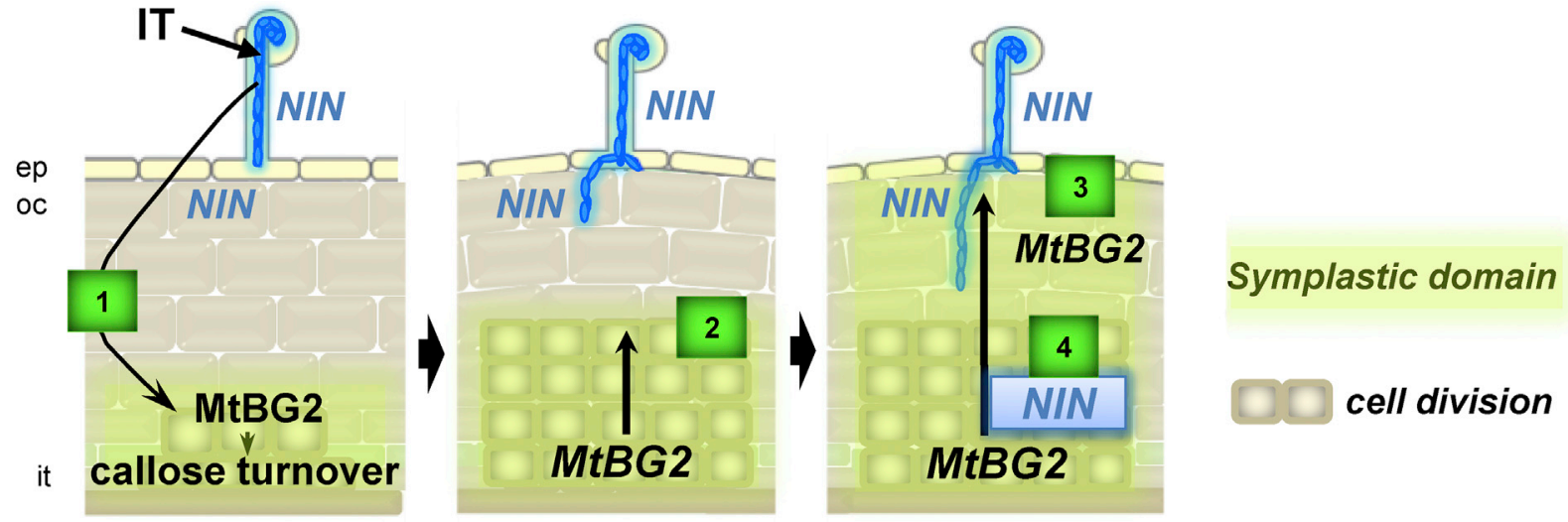

1 Rhizobia-induced signaling triggers MtBG2 expression and callose degradation in the inner tissues

2 MtBG2 mediates the generation of symplastic connections in dividing nodule primordia

3 Outwards expression of MtBG2 creates symplastic connections in root cells preparing or undergoing infection

4 Newly-formed symplastic domains are required for the expression of NIN in inner tissues

Figure 7. Schematic Model for the Role of MtBG2 and Symplastic Communication during Root Nodule Development in M. truncatula The construction of rhizobial infection threads involves cell-autonomous signaling in the root epidermis (ep) and outer cortex (oc), which results in the local expression of the transcription factors NIN and NF-YA1 (Figure 6; 1). In parallel, unknown non-cell-autonomous signaling pathways induce the expression of MtBG2, and possibly other functionally related $\beta-1,3$ glucanases, in the inner pericycle and cortical root tissues (it) (Figure 1). These enzymes are responsible for callose degradation (turnover) at PD sites and the regulation of symplastic intercellular transport (Figure 3). The expression of MtBG2 likely contributes to symplastic communication between the phloem and the inner cortex, which correlates with mitotic cell divisions and the formation of the nodule primordia (2) [34]. MtBG2 expression subsequently expands toward outer tissues accompanying the entry of rhizobial infection threads (Figure 1), where callose degradation, mediated by MtBG2, contributes to the creation of symplastic domains (Figures 2, 3, 4, and 5; 3). This symplastic regulated pathway is required for the activation of NIN and NF-YA1 in the middle/inner cortex (Figure 6), which are necessary for the proper regulation of nodule development and colonization (4).

callose accumulation and to block symplastic connections in Arabidopsis roots [37, 38, 44, 45]. Expression of cals3m in M. truncatula under the control of symbiotic promoters ( $p M t B G 2$, $p E R N 1$, and $p A N N 1$ ) affects root nodule development and colonization (Figures 4 and 5). A root nodulation phenotype was also observed when RNAi constructs, which target MtBG2 expression, were expressed under the ERN1 promoter, and an increase in nodulation frequency, mimicking the ectopic expression of MtBG2 (Figure 3), was observed when PdBG1 was expressed under the same promoter. Although root hair infection thread for- mation was favored in roots expressing PdBG1 or MtBG2, no major phenotypic effect was observed in roots expressing cals $3 m$ in the root epidermis (whether driven by MtBG2, ERN1, $A N N 1$, or EXPA promoters), suggesting that symplastic connectivity is not critical for infection thread initiation. Accordingly, the tissue-specific expression of NIN and NF-YA1 in the epidermis and outer cortex underlying a growing infection thread was not strongly affected in the cals $3 m$ context (Figures 6 and S7). This indicates that the machinery required for the local construction of infection threads, orchestrated by ERN1 and NIN,

transcripts were also analyzed in cals $3 \mathrm{~m}$ or GFP control roots 3 and 7 dpi with rhizobia. Boxplots in $(A)-(D)$ represent the average values of individual plants $(n=24$ for GFP; $\mathrm{n}=24$ for $c a / s 3 m$ ) from three independent biological experiments after normalization against three reference transcripts. Boxplots represent first and third quartile (horizontal box sides) and minimum and maximum (outside whiskers). Central lines refer to median, and black circles depict mean values. Twosample t test of the values revealed statistically significant differences ( ${ }^{\star \star *} \mathrm{p}<0.001$ ) between GFP and cals $3 m$ samples for NIN in (C) and NF-YA1 in (D). AspinWelch two-sample $t$ test of the values revealed statistically significant differences $\left({ }^{*} \mathrm{p}<0.05\right)$ between GFP and cals3m samples for ERN1 in $(\mathrm{A})$.

(E-P) Representative images of $M$. truncatula roots expressing a 1.4-kb pNIN:GUS (E-J), a 2-kb pNIN:GUS (K-P), or co-expressing the respective fusions with pERN1:cals3m (H-J or N-P) during early stages of nodule primordia development and infection. In control pNIN:GUS roots, GUS activity is first detected in outer epidermal and cortical tissues associated with infection sites (K). At a subsequent stage, GUS activity is associated with both epidermal and cortical tissues undergoing infection (black arrowheads) and inner dividing cortical tissues (white arrowheads), as illustrated in (E)-(G) and in Ruthenium-Red-stained longitudinal sections ( $\mathrm{L}$ and $\mathrm{M}$ ). In pNIN:GUS/pERN1:cals3m ( $\mathrm{H}-\mathrm{J}$ and $\mathrm{N}-\mathrm{P}$ ) roots, GUS activity is limited to outer tissues (black arrowheads in $\mathrm{H}-\mathrm{J}$ and in longitudinal Ruthenium Red sections in N-P) around growing infection threads (arrows) and no longer detected in inner cortical tissues (asterisk in O). The expression of the pNIN:GUS fusion was observed in M. truncatula roots 3-7 dpi with rhizobia ( $\mathrm{n}=59$ for $1.4 \mathrm{~kb} p N I N: G U S, \mathrm{n}=40$ for $1.4 \mathrm{~kb} p N I N: G U S / p E R N 1: c a l s 3 m, \mathrm{n}=36$ for $2.0 \mathrm{~kb}$ pNIN:GUS, and $\mathrm{n}=24$ for $2.0 \mathrm{~kb}$ pNIN:GUS/pERN1:cals3m). Representative images were taken $4 \mathrm{dpi}(\mathrm{E}-\mathrm{G})$ and $7 \mathrm{dpi}(\mathrm{H}-\mathrm{P})$ with rhizobia. c, cortex; ep, epidermis. Scale bars represent $50 \mu \mathrm{m}$.

See also Figures S6 and S7. 
is cell-autonomously regulated, which is consistent with the described mechanisms for cell-autonomous control of infection by symbiotic signaling $[25,46]$.

Our work and other studies [25, 46] suggest that non-cellautonomous signal(s) exist to integrate the epidermal and cortical nodulation programs, but the nature of this signal(s) or whether it is symplastically regulated is currently unknown. Blocking symplastic connectivity using $p E R N 1$ :cals3m led over time to a drastic reduction in the expression of NIN and the associated target NF-YA1 in the cortex of the developing nodule primordia (Figures 6, S6, and S7). This implies that the transmission of a putative non-cell-autonomous signal to regulate NIN expression in dividing nodule primordia tissues is symplastically regulated. MtCRE1, which is interconnected with NIN during cytokinin-mediated nodule organogenesis in the cortex [5], does not have its expression modified in the pERN1:cals $3 m$ context. This suggests that the putative symplastic signal regulating NIN expression in the nodule primordia operates downstream or independent of CRE1.

Previous studies demonstrated the importance of NIN and other key regulators in the control of both infection in the epidermis and nodule organogenesis in the root cortex [22, 25, 30]. NIN in particular has the ability to induce nodule organogenesis in the cortex when expressed exclusively in the root epidermis of M. truncatula [30], which has led to the proposition that NIN itself or a downstream signal might move from the epidermis to the cortex to induce nodule organogenesis. We demonstrated here that both nodule development and colonization were affected in pMtBG2:cals3m/pERN1:cal3sm/pANN1:cal3sm roots (Figures 4 and 5), implying that signals required for these processes are, at least in part, dependent on symplastic communication. Although nodule formation was affected, poorly colonized nodule primordia/nodules were formed in cals $3 m$ roots, suggesting that signal(s) from the epidermis could be still partially transmitted to induce cell division in the cortex. This suggests that the putative organogenesis-triggering signal does not absolutely require a symplastic route to be transmitted and might navigate through symplastic, apoplastic, and/or transcellular paths. Cytokinins or flavonoids synthesized in the root epidermis of $M$. truncatula are potential candidates for this non-cell-autonomous role that rapidly promotes cell division in the cortex [5, 47, 48]. However, in pMtBG2:cals3m, pERN1:cals3m, and pANN1:cals3m situations, a more pronounced phenotype was observed during nodule colonization, giving rise to nodule primordia with infection arrests or under-differentiated nodules with signs of defective bacterial release into plant cells. These results are consistent with the enhanced nodule colonization observed in pUBIQ:MtBG2 and pERN1:PdBG1 roots, suggesting the existence of a secondary symplastically transmitted signal required for the later nodule primordia colonization and the expression of NIN in the cortex.

Symplastic routes mediate the transport of a number of noncell-autonomous molecules, including mobile RNAs and transcription factors that play critical roles in the regulation of most processes related to organ development in plants [36, 49]. NIN transcription was shown to be regulated by the CYCLOPS/ CCaMK complex [17] and potentially by the GRAS-type NSP1/ NSP2 complex together with the IPN2 MYB factor [20, 50]. Members of the GRAS and the MYB families of transcription factors have the ability to move intercellularly, presumably through PD
[44]. However, no evidence has been provided to date supporting the function of these regulators as mobile signals in legumes and/or during symbiosis. Future research will help to elucidate the identity of the mobile factors involved in nodulation and to establish the molecular mechanism and crosstalk with other cell-autonomous pathways.

\section{STAR $\star$ METHODS}

Detailed methods are provided in the online version of this paper and include the following:

- KEY RESOURCES TABLE

- CONTACT FOR REAGENT AND RESOURCE SHARING

- EXPERIMENTAL MODEL AND SUBJECT DETAILS

O Plant material, bacterial strains and growth conditions

O DNA Constructs

- METHOD DETAILS

- Generation and selection of $M$. truncatula transgenic roots

Growth conditions and inoculation of M. truncatula roots

$\bigcirc$ GUS and $\beta$-Galactosidase assays

O Symplastic transport assays

o Callose immunolocalization

O Microscopy methods

$O$ RNA extraction and QRT-PCR analyses

Phylogenetic analysis

- QUANTIFICATION AND STATISTICAL ANALYSIS

- DATA AND SOFTWARE AVAILABILITY

\section{SUPPLEMENTAL INFORMATION}

Supplemental Information includes seven figures and two tables and can be found with this article online at https://doi.org/10.1016/j.cub.2018.09.031.

\section{ACKNOWLEDGMENTS}

We thank D. Barker, J. Fournier, and P. Gamas for critical reading of the manuscript; A. Kelner and M.-F. Jardinaud for valuable advices for statistical analyses using the R software; the TRI-FRAIB miscroscopy platform and A. Lepage for technical assistance with the Technovit embedding; Ykä Helariutta (Sainsbury Laboratory, Cambridge, UK) for kindly providing the cals $3 m$ construct; J. Fournier for providing the Sm 2011-mCherry strain; and C. Rosenberg for providing the pCambia goldengate vectors. R.G.-P. was supported by Leeds University 110 scholarship, M.B. was supported by the French Laboratoire d'Excellence (Labex) TULIP post-doctoral grant "Young Scientists for the Future" and by an AgreenSkills fellowship (no. 2013609398). C.R. is a postdoctoral fellow from the ANR NODCCAAT (ANR-15CE20-0012-01) and INRA Agreenskills (no. 2010-267196). P.K. was supported by a BBSRC DTP (BB/M011151/1). Y.B.-A. lab work was supported by research grants from the Royal Society, EPSRC (EF/M027740/1), and the Leverhulme Trust grant RPG-2016-136, and F.d.C.-N. lab work was supported by ANR COME-IN (no. ANR-14-CE35-0007-01) and TULIP (no. ANR-10-LABX41) grants.

\section{AUTHOR CONTRIBUTIONS}

M.B., Y.B.-A., and F.d.C.-N. conceived the project. The Leeds team headed by Y.B.-A. designed and performed callose and $\beta-1,3$-glucanase immunolocalization (R.G.-P. and P.K.), phylogenetic analyses (R.G.-P. and Y.B.-A.), MtBG2/PdBG1 expression (R.G.-P. and P.K.), and GFP symplastic transport experiments (P.K. and Y.B.-A.), with contributions of G.E.D.O. for experimental 
design. The LIPM team headed by F.d.C.-N. designed and performed CFDA and YFP symplastic transport (M.B.), calsm3 and RNAi experiments (L.F., M.B., and F.d.C.-N.), MtBG2 and NIN/NF-YA1 expression (L.F., M.B., and F.d.C.-N.), and qRT-PCR and statistical analyses (L.F.), with contributions of C.R. and A.N., who provided cDNAs for MtBGs qRT-PCR. Y.B.-A. and F.d.C.-N. are co-responsible for the work and wrote the manuscript.

\section{DECLARATION OF INTERESTS}

The authors declare no competing interests.

Received: January 17, 2018

Revised: July 27, 2018

Accepted: September 13, 2018

Published: November 8, 2018

\section{REFERENCES}

1. Oldroyd, G.E. (2013). Speak, friend, and enter: signalling systems that promote beneficial symbiotic associations in plants. Nat. Rev. Microbiol. 11, 252-263.

2. Fournier, J., Teillet, A., Chabaud, M., Ivanov, S., Genre, A., Limpens, E., de Carvalho-Niebel, F., and Barker, D.G. (2015). Remodeling of the infection chamber before infection thread formation reveals a two-step mechanism for rhizobial entry into the host legume root hair. Plant Physiol. 167, 12331242.

3. Xiao, T.T., Schilderink, S., Moling, S., Deinum, E.E., Kondorosi, E., Franssen, H., Kulikova, O., Niebel, A., and Bisseling, T. (2014). Fate map of Medicago truncatula root nodules. Development 141, 3517-3528.

4. Ferguson, B.J., Mens, C., Hastwell, A.H., Zhang, M., Su, H., Jones, C.H., Chu, X., and Gresshoff, P.M. (2018). Legume nodulation: the host controls the party. Plant Cell Environ. Published online May 28, 2018. https://doi. org/10.1111/pce.13348.

5. Gamas, P., Brault, M., Jardinaud, M.F., and Frugier, F. (2017). Cytokinins in Symbiotic Nodulation: When, Where, What For? Trends Plant Sci. 22, 792-802.

6. Bensmihen, S. (2015). Hormonal Control of Lateral Root and Nodule Development in Legumes. Plants (Basel) 4, 523-547.

7. Zipfel, C., and Oldroyd, G.E. (2017). Plant signalling in symbiosis and immunity. Nature 543, 328-336.

8. Lévy, J., Bres, C., Geurts, R., Chalhoub, B., Kulikova, O., Duc, G., Journet, E.P., Ané, J.M., Lauber, E., Bisseling, T., et al. (2004). A putative Ca2+ and calmodulin-dependent protein kinase required for bacterial and fungal symbioses. Science 303, 1361-1364.

9. Tirichine, L., Imaizumi-Anraku, H., Yoshida, S., Murakami, Y., Madsen, L.H., Miwa, H., Nakagawa, T., Sandal, N., Albrektsen, A.S., Kawaguchi, M., et al. (2006). Deregulation of a Ca2+/calmodulin-dependent kinase leads to spontaneous nodule development. Nature 441, 1153-1156.

10. Marsh, J.F., Rakocevic, A., Mitra, R.M., Brocard, L., Sun, J., Eschstruth, A., Long, S.R., Schultze, M., Ratet, P., and Oldroyd, G.E. (2007). Medicago truncatula NIN is essential for rhizobial-independent nodule organogenesis induced by autoactive calcium/calmodulin-dependent protein kinase. Plant Physiol. 144, 324-335.

11. Schauser, L., Roussis, A., Stiller, J., and Stougaard, J. (1999). A plant regulator controlling development of symbiotic root nodules. Nature 402, 191-195.

12. Middleton, P.H., Jakab, J., Penmetsa, R.V., Starker, C.G., Doll, J., Kaló, P., Prabhu, R., Marsh, J.F., Mitra, R.M., Kereszt, A., et al. (2007). An ERF transcription factor in Medicago truncatula that is essential for Nod factor signal transduction. Plant Cell 19, 1221-1234.

13. Andriankaja, A., Boisson-Dernier, A., Frances, L., Sauviac, L., Jauneau, A., Barker, D.G., and de Carvalho-Niebel, F. (2007). AP2-ERF transcription factors mediate Nod factor dependent Mt ENOD11 activation in root hairs via a novel cis-regulatory motif. Plant Cell 19, 2866-2885.
14. Cerri, M.R., Wang, Q., Stolz, P., Folgmann, J., Frances, L., Katzer, K., Li X., Heckmann, A.B., Wang, T.L., Downie, J.A., et al. (2017). The ERN1 transcription factor gene is a target of the CCaMK/CYCLOPS complex and controls rhizobial infection in Lotus japonicus. New Phytol. 215, 323-337.

15. Fonouni-Farde, C., Tan, S., Baudin, M., Brault, M., Wen, J., Mysore, K.S. Niebel, A., Frugier, F., and Diet, A. (2016). DELLA-mediated gibberellin signalling regulates Nod factor signalling and rhizobial infection. Nat. Commun. 7, 12636.

16. Jin, Y., Liu, H., Luo, D., Yu, N., Dong, W., Wang, C., Zhang, X., Dai, H., Yang, J., and Wang, E. (2016). DELLA proteins are common components of symbiotic rhizobial and mycorrhizal signalling pathways. Nat. Commun. 7, 12433.

17. Singh, S., Katzer, K., Lambert, J., Cerri, M., and Parniske, M. (2014). CYCLOPS, a DNA-binding transcriptional activator, orchestrates symbiotic root nodule development. Cell Host Microbe 15, 139-152.

18. Cerri, M.R., Frances, L., Laloum, T., Auriac, M.C., Niebel, A., Oldroyd, G.E., Barker, D.G., Fournier, J., and de Carvalho-Niebel, F. (2012). Medicago truncatula ERN transcription factors: regulatory interplay with NSP1/NSP2 GRAS factors and expression dynamics throughout rhizobial infection. Plant Physiol. 160, 2155-2172.

19. Laloum, T., Baudin, M., Frances, L., Lepage, A., Billault-Penneteau, B. Cerri, M.R., Ariel, F., Jardinaud, M.F., Gamas, P., de Carvalho-Niebel, F., and Niebel, A. (2014). Two CCAAT-box-binding transcription factors redundantly regulate early steps of the legume-rhizobia endosymbiosis. Plant J. 79, 757-768.

20. Hirsch, S., Kim, J., Muñoz, A., Heckmann, A.B., Downie, J.A., and Oldroyd, G.E. (2009). GRAS proteins form a DNA binding complex to induce gene expression during nodulation signaling in Medicago truncatula. Plant Cell 21, 545-557.

21. Xie, F., Murray, J.D., Kim, J., Heckmann, A.B., Edwards, A., Oldroyd, G.E., and Downie, J.A. (2012). Legume pectate lyase required for root infection by rhizobia. Proc. Natl. Acad. Sci. USA 109, 633-638.

22. Cerri, M.R., Frances, L., Kelner, A., Fournier, J., Middleton, P.H., Auriac, M.C., Mysore, K.S., Wen, J., Erard, M., Barker, D.G., et al. (2016). The symbiosis-related ERN transcription factors act in concert to coordinate rhizobial host root infection. Plant Physiol. 171, 1037-1054.

23. Kawaharada, Y., James, E.K., Kelly, S., Sandal, N., and Stougaard, J. (2017). The Ethylene Responsive Factor Required for Nodulation 1 (ERN1) Transcription Factor Is Required for Infection-Thread Formation in Lotus japonicus. Mol. Plant Microbe Interact. 30, 194-204.

24. Yano, K., Aoki, S., Liu, M., Umehara, Y., Suganuma, N., Iwasaki, W., Sato, S., Soyano, T., Kouchi, H., and Kawaguchi, M. (2017). Function and evoIution of a Lotus japonicus AP2/ERF family transcription factor that is required for development of infection threads. DNA Res. 24, 193-203.

25. Madsen, L.H., Tirichine, L., Jurkiewicz, A., Sullivan, J.T., Heckmann, A.B., Bek, A.S., Ronson, C.W., James, E.K., and Stougaard, J. (2010). The molecular network governing nodule organogenesis and infection in the model legume Lotus japonicus. Nat. Commun. 1, 10.

26. Griesmann, M., Chang, Y., Liu, X., Song, Y., Haberer, G., Crook, M.B. Billault-Penneteau, B., Lauressergues, D., Keller, J., Imanishi, L., et al. (2018). Phylogenomics reveals multiple losses of nitrogen-fixing root nodule symbiosis. Science 361 , eaat 1743 .

27. van Velzen, R., Holmer, R., Bu, F., Rutten, L., van Zeijl, A., Liu, W., Santuari, L., Cao, Q., Sharma, T., Shen, D., et al. (2018). Comparative genomics of the nonlegume Parasponia reveals insights into evolution of nitrogen-fixing rhizobium symbioses. Proc. Natl. Acad. Sci. USA 115, E4700-E4709.

28. Soyano, T., Kouchi, H., Hirota, A., and Hayashi, M. (2013). Nodule inception directly targets NF-Y subunit genes to regulate essential processes of root nodule development in Lotus japonicus. PLoS Genet. 9, e1003352.

29. Kawaharada, Y., Nielsen, M.W., Kelly, S., James, E.K., Andersen, K.R., Rasmussen, S.R., Füchtbauer, W., Madsen, L.H., Heckmann, A.B., Radutoiu, S., and Stougaard, J. (2017). Differential regulation of the Epr3 receptor coordinates membrane-restricted rhizobial colonization of root nodule primordia. Nat. Commun. 8, 14534 
30. Vernié, T., Kim, J., Frances, L., Ding, Y., Sun, J., Guan, D., Niebel, A., Gifford, M.L., de Carvalho-Niebel, F., and Oldroyd, G.E.D. (2015). The NIN Transcription Factor Coordinates Diverse Nodulation Programs in Different Tissues of the Medicago truncatula Root. Plant Cell 27, 34103424.

31. Yoro, E., Suzaki, T., Toyokura, K., Miyazawa, H., Fukaki, H., and Kawaguchi, M. (2014). A Positive Regulator of Nodule Organogenesis, NODULE INCEPTION, Acts as a Negative Regulator of Rhizobial Infection in Lotus japonicus. Plant Physiol. 165, 747-758.

32. Mortier, V., Den Herder, G., Whitford, R., Van de Velde, W., Rombauts, S., D'Haeseleer, K., Holsters, M., and Goormachtig, S. (2010). CLE peptides control Medicago truncatula nodulation locally and systemically. Plant Physiol. 153, 222-237.

33. Okamoto, S., Ohnishi, E., Sato, S., Takahashi, H., Nakazono, M., Tabata, S., and Kawaguchi, M. (2009). Nod factor/nitrate-induced CLE genes that drive HAR1-mediated systemic regulation of nodulation. Plant Cell Physiol. 50, 67-77.

34. Complainville, A., Brocard, L., Roberts, I., Dax, E., Sever, N., Sauer, N., Kondorosi, A., Wolf, S., Oparka, K., and Crespi, M. (2003). Nodule initiation involves the creation of a new symplasmic field in specific root cells of medicago species. Plant Cell 15, 2778-2791.

35. Tilsner, J., Nicolas, W., Rosado, A., and Bayer, E.M. (2016). Staying Tight: Plasmodesmal Membrane Contact Sites and the Control of Cell-to-Cell Connectivity in Plants. Annu. Rev. Plant Biol. 67, 337-364.

36. Otero, S., Helariutta, Y., and Benitez-Alfonso, Y. (2016). Symplastic communication in organ formation and tissue patterning. Curr. Opin. Plant Biol. 29, 21-28.

37. Vatén, A., Dettmer, J., Wu, S., Stierhof, Y.D., Miyashima, S., Yadav, S.R., Roberts, C.J., Campilho, A., Bulone, V., Lichtenberger, R., et al. (2011). Callose biosynthesis regulates symplastic trafficking during root development. Dev. Cell 21, 1144-1155.

38. Benitez-Alfonso, Y., Faulkner, C., Pendle, A., Miyashima, S., Helariutta, Y., and Maule, A. (2013). Symplastic intercellular connectivity regulates lateral root patterning. Dev. Cell 26, 136-147.

39. Gaudioso-Pedraza, R., and Benitez-Alfonso, Y. (2014). A phylogenetic approach to study the origin and evolution of plasmodesmata-localized glycosyl hydrolases family 17. Front. Plant Sci. 5, 212.

40. De Carvalho-Niebel, F., Timmers, A.C., Chabaud, M., Defaux-Petras, A., and Barker, D.G. (2002). The Nod factor-elicited annexin MtAnn1 is preferentially localised at the nuclear periphery in symbiotically activated root tissues of Medicago truncatula. Plant J. 32, 343-352.

41. Ovchinnikova, E., Journet, E.P., Chabaud, M., Cosson, V., Ratet, P., Duc, G., Fedorova, E., Liu, W., den Camp, R.O., Zhukov, V., et al. (2011). IPD3 controls the formation of nitrogen-fixing symbiosomes in pea and Medicago Spp. Mol. Plant Microbe Interact. 24, 1333-1344.

42. Laporte, P., Lepage, A., Fournier, J., Catrice, O., Moreau, S., Jardinaud, M.F., Mun, J.H., Larrainzar, E., Cook, D.R., Gamas, P., and Niebel, A. (2014). The CCAAT box-binding transcription factor NF-YA1 controls rhizobial infection. J. Exp. Bot. 65, 481-494.

43. Plet, J., Wasson, A., Ariel, F., Le Signor, C., Baker, D., Mathesius, U., Crespi, M., and Frugier, F. (2011). MtCRE1-dependent cytokinin signaling integrates bacterial and plant cues to coordinate symbiotic nodule organogenesis in Medicago truncatula. Plant J. 65, 622-633.

44. Liu, Y., Xu, M., Liang, N., Zheng, Y., Yu, Q., and Wu, S. (2017). Symplastic communication spatially directs local auxin biosynthesis to maintain root stem cell niche in Arabidopsis. Proc. Natl. Acad. Sci. USA 114, 4005-4010.

45. Wu, S., O’Lexy, R., Xu, M., Sang, Y., Chen, X., Yu, Q., and Gallagher, K.L. (2016). Symplastic signaling instructs cell division, cell expansion, and cell polarity in the ground tissue of Arabidopsis thaliana roots. Proc. Natl. Acad. Sci. USA 113, 11621-11626.

46. Rival, P., de Billy, F., Bono, J.J., Gough, C., Rosenberg, C., and Bensmihen, S. (2012). Epidermal and cortical roles of NFP and DMI3 in coordinating early steps of nodulation in Medicago truncatula. Development 139, 3383-3391.
47. Breakspear, A., Liu, C., Roy, S., Stacey, N., Rogers, C., Trick, M., Morieri, G., Mysore, K.S., Wen, J., Oldroyd, G.E., et al. (2014). The root hair "infectome" of Medicago truncatula uncovers changes in cell cycle genes and reveals a requirement for Auxin signaling in rhizobial infection. Plant Cell $26,4680-4701$.

48. Deinum, E.E., Kohlen, W., and Geurts, R. (2016). Quantitative modelling of legume root nodule primordium induction by a diffusive signal of epidermal origin that inhibits auxin efflux. BMC Plant Biol. 16, 254.

49. Han, X., Kumar, D., Chen, H., Wu, S., and Kim, J.Y. (2014). Transcription factor-mediated cell-to-cell signalling in plants. J. Exp. Bot. 65, 17371749.

50. Kang, H., Chu, X., Wang, C., Xiao, A., Zhu, H., Yuan, S., Yang, Z., Ke, D., Xiao, S., Hong, Z., and Zhang, Z. (2014). A MYB coiled-coil transcription factor interacts with NSP2 and is involved in nodulation in Lotus japonicus. New Phytol. 201, 837-849.

51. Quandt, H.J., Puhler, A., and Broer, I. (1993). Transgenic root nodules of Vicia hirsuta: a fast and efficient system for the study of gene expression in indeterminate-type nodules. Mol. Plant Microbe Interact. 6, 699-706.

52. Ardourel, M., Demont, N., Debellé, F., Maillet, F., de Billy, F., Promé, J.C., Dénarié, J., and Truchet, G. (1994). Rhizobium meliloti lipooligosaccharide nodulation factors: different structural requirements for bacterial entry into target root hair cells and induction of plant symbiotic developmental responses. Plant Cell 6, 1357-1374.

53. Fournier, J., Timmers, A.C., Sieberer, B.J., Jauneau, A., Chabaud, M., and Barker, D.G. (2008). Mechanism of infection thread elongation in root hairs of Medicago truncatula and dynamic interplay with associated rhizobial colonization. Plant Physiol. 148, 1985-1995.

54. Rodrigues, M.A., Gomes, D.A., Leite, M.F., Grant, W., Zhang, L., Lam, W., Cheng, Y.C., Bennett, A.M., and Nathanson, M.H. (2007). Nucleoplasmic calcium is required for cell proliferation. J. Biol. Chem. 282, 17061-17068.

55. Fliegmann, J., Jauneau, A., Pichereaux, C., Rosenberg, C., Gasciolli, V., Timmers, A.C., Burlet-Schiltz, O., Cullimore, J., and Bono, J.J. (2016). LYR3, a high-affinity LCO-binding protein of Medicago truncatula, interacts with LYK3, a key symbiotic receptor. FEBS Lett. 590, 1477-1487.

56. Karimi, M., Inzé, D., and Depicker, A. (2002). GATEWAY vectors for Agrobacterium-mediated plant transformation. Trends Plant Sci. 7, 193-195.

57. Schneider, C.A., Rasband, W.S., and Eliceiri, K.W. (2012). NIH Image to ImageJ: 25 years of image analysis. Nat. Methods 9, 671-675.

58. Stöver, B.C., and Müller, K.F. (2010). TreeGraph 2: combining and visualizing evidence from different phylogenetic analyses. BMC Bioinformatics 11, 7.

59. Cosson, V., Durand, P., d'Erfurth, I., Kondorosi, A., and Ratet, P. (2006). Medicago truncatula transformation using leaf explants. Methods Mol. Biol. 343, 115-127.

60. Engler, C., and Marillonnet, S. (2014). Golden Gate cloning. Methods Mol. Biol. 1116, 119-131.

61. Boisson-Dernier, A., Chabaud, M., Garcia, F., Bécard, G., Rosenberg, C., and Barker, D.G. (2001). Agrobacterium rhizogenes-transformed roots of Medicago truncatula for the study of nitrogen-fixing and endomycorrhizal symbiotic associations. Mol. Plant Microbe Interact. 14, 695-700.

62. Boisson-Dernier, A., Andriankaja, A., Chabaud, M., Niebel, A., Journet, E.P., Barker, D.G., and de Carvalho-Niebel, F. (2005). MtENOD11 gene activation during rhizobial infection and mycorrhizal arbuscule development requires a common AT-rich-containing regulatory sequence. Mol. Plant Microbe Interact. 18, 1269-1276.

63. Pendle, A., and Benitez-Alfonso, Y. (2015). Immunofluorescence detection of callose deposition around plasmodesmata sites. Methods Mol. Biol. 1217, 95-104.

64. Roux, B., Rodde, N., Jardinaud, M.F., Timmers, T., Sauviac, L., Cottret, L., Carrère, S., Sallet, E., Courcelle, E., Moreau, S., et al. (2014). An integrated analysis of plant and bacterial gene expression in symbiotic root nodules using laser-capture microdissection coupled to RNA sequencing. Plant J. $77,817-837$. 


\section{STAR $\star$ METHODS}

\section{KEY RESOURCES TABLE}

\begin{tabular}{|c|c|c|}
\hline REAGENT or RESOURCE & SOURCE & IDENTIFIER \\
\hline \multicolumn{3}{|l|}{ Antibodies } \\
\hline Anti-callose: (1-3)-beta-glucan-directed monoclonal antibody & Biosupplies & $\begin{array}{l}\text { http://www.biosupplies.com.au/docs/ } \\
\text { 400-2.pdf } \\
\text { Cat\#400-2; RRID:AB_2747399 }\end{array}$ \\
\hline \multicolumn{3}{|l|}{ Experimental Models/Strains } \\
\hline Medicago truncatula A17 & $\mathrm{N} / \mathrm{A}$ & $\begin{array}{l}\text { http://www.noble.org/ } \\
\text { medicagohandbook }\end{array}$ \\
\hline Agrobacterium rhizogenes ARquA1 & [51] & N/A \\
\hline Sinorhizobium meliloti 1021-lacZ (with the pXLGD4 lacZ reporter) & [47] & Sm 1021-lacZ \\
\hline Sinorhizobium meliloti (constitutively expressing a hemA-lacZ fusion) & [52] & Sm 2011-lacZ \\
\hline Sinorhizobium meliloti Sm 2011-CFP (constitutively expressing CFP) & [53] & Sm 2011-CFP \\
\hline $\begin{array}{l}\text { Sinorhizobium meliloti Sm 2011-mCherry (constitutively expressing } \\
\text { mCherry) }\end{array}$ & N/A & $\begin{array}{l}\text { Sm } 2011-m \text { Cherry Kindly provided } \\
\text { by J. Fournier, LIPM. }\end{array}$ \\
\hline \multicolumn{3}{|l|}{ Critical Commercial Assays } \\
\hline Gateway BP and LR clonase II enzyme mixes & Invitrogen & $\begin{array}{l}\text { Cat\#11789-100 and Cat\#11791-100, } \\
\text { respectively }\end{array}$ \\
\hline Bsa I enzyme for Goldengate reactions & New England BIOLABS & Cat\#R0535S \\
\hline $\begin{array}{l}\text { X-Gluc (5-bromo-4-chloro-3-indoxyl-b-D-GlcA, } \\
\text { cyclohexylammonium salt }\end{array}$ & Biosynth & Cat\#B7300; CAS: 114162-64-0 \\
\hline $\begin{array}{l}\text { Magenta-Gal substrate (5-bromo-6-chloro-3-indoxyl-b-D- } \\
\text { galactopyranoside }\end{array}$ & Biosynth & Cat\#B7200; CAS: 93863-88-8 \\
\hline X-Gal substrate (5-bromo-4-chloro-3-indolyl-b-Dgalactopyranoside & Fisher BioReagents & Cat\#10113253; CAS: 7240-90-6 \\
\hline Technovit 7100 & Heraeus-Kulzer, Germany & Cat\#64709003 \\
\hline Macherey-Nagel NUCLEOSPIN RNA kit & Macherey-Nagel & Cat\#740955.250 \\
\hline Agilent RNA Nano Chip and Reagents & Agilent Technologies & Cat\#5067-1511 \\
\hline Transcriptor Reverse Transcriptase for QRT-PCR & Roche & Cat\#03531287001 \\
\hline LightCycler480 Sybr Green I Master & Roche & Cat\#04707516001 \\
\hline Anilin Blue & EMS & Cat\#10840; CAS: $28631-66-5$ \\
\hline glutaraldehyde $25 \%$ & EMS & Cat\#16210; CAS: 111-30-8 \\
\hline Paraformaldehyde aqueous solution $32 \%$ & EMS & Cat\#15714; CAS: $30525-89-4$ \\
\hline 5(6)-Carboxyfluorescein diacetate (CFDA) & Sigma-Aldrich & Cat\#21879; CAS: 124387-19-5 \\
\hline Ruthenium red & Sigma-Aldrich & Cat\#R-2751; CAS: 11103-72-3 \\
\hline \multicolumn{3}{|l|}{ Recombinant DNA } \\
\hline PdBG1(At3g13560)-eGFP protein fusion & GenScript, this work & PdBG1-GFP \\
\hline MtBG2 (Medtr3g083580)-mCherry protein fusion & GenScript, this work & MtBG2-mCherry \\
\hline pMtBG2:GUS in a pCambiaCR1 derived vector & This work & pMtBG2:GUS \\
\hline 1.4 kb pNIN:GUS reporter in a pCambiaCR1 derived vector & This work & $1.4 \mathrm{~kb} p$ NIN:GUS \\
\hline $2.1 \mathrm{~kb}$ pNIN:GUS reporter in a pCambiaCR1 derived vector & This work & $2.1 \mathrm{~kb} p$ NIN:GUS \\
\hline $2.2 \mathrm{~kb}$ pERN1:GUS reporter in a pCambiaCR1 derived vector & This work & pERN1:GUS \\
\hline 2.2Kb pERN1-MtBG2 RNAi-5'UTR in a pCambiaCR1 derived vector & This work & MtBG2 RNAi-5' \\
\hline $\begin{array}{l}2.2 K b \text { pERN1-MtBG2 RNAi-cds(850bp) in a pCambiaCR1 derived } \\
\text { vector }\end{array}$ & This work & MtBG2 RNAi-cds \\
\hline 2.2 Kb pNFYA1:GUS in a pK7m34GW vector & [42] & pNF-YA1:GUS \\
\hline pEXPA:YFP in a pCambiaCR1 derived vector & [30] and this work & PEXPA:YFP \\
\hline$p E X P A: m C h e r r y-E R$ in a pCambiaCR1 derived vector & [30] and this work & pEXPA:mCherry-ER \\
\hline pEXPA:cals3m in a pK7WG2-R derived vector & This work & pEXPA:cals $3 m$ \\
\hline
\end{tabular}




\begin{tabular}{|c|c|c|}
\hline \multicolumn{3}{|l|}{ Continued } \\
\hline REAGENT or RESOURCE & SOURCE & IDENTIFIER \\
\hline$p E X P A: P V_{c d}\left(P V_{c d}\right.$, parvalbumin mutated) :NLS:CFP control & [54] & $\begin{array}{l}\text { Addgene\#16345, modified in } \\
\text { this work }\end{array}$ \\
\hline pERN1:cals3m in a pK7WG2-R derived vector & This work & pERN1:cals3m \\
\hline pERN1:GFP in a pK7WG2-R derived vector & This work & pERN1:GFP \\
\hline pMtBG2:cals3m in a pK7WG2-R derived vector & This work & pMtBG2:cals3m \\
\hline pMtBG2:GFP in a pK7WG2-R derived vector & This work & pMtBG2:GFP \\
\hline pANN1:cals3m in a pK7WG2 derived vector & This work & pANN1:cals3m \\
\hline pANN1:GFP in a pK7WG2 derived vector & This work & pANN1:GFP \\
\hline pDONR221-cals3m & $\begin{array}{l}\text { [37], kindly provided } \\
\text { by Ykä Helariutta }\end{array}$ & pDONR221-cals3m \\
\hline pCambiaCR1 vectors & [55] & N/A \\
\hline pK7WG2-R-pLjUb vector & [56] & N/A \\
\hline \multicolumn{3}{|l|}{ Oligonucleotides } \\
\hline See Table S2 for the List of primers used in this study. & This work & N/A \\
\hline \multicolumn{3}{|l|}{ Deposited Data } \\
\hline Raw and analyzed data & This work & $\begin{array}{l}\text { Medeley database; https://doi.org/ } \\
\text { 10.17632/5hzcxdb4dp.1 }\end{array}$ \\
\hline \multicolumn{3}{|l|}{ Software and Algorithms } \\
\hline Leica, ImageJ & [57] & http://imagej.nih.gov/ij/ \\
\hline Volocity version 6.0 .1 software & PerkinElmer & License $\mathrm{n}^{\circ}$ 147.99.102.74 \\
\hline Figtree & $\begin{array}{l}\text { http://tree.bio.ed.ac.uk/ } \\
\text { software/figtree/ }\end{array}$ & $\mathrm{N} / \mathrm{A}$ \\
\hline TreeGraph2 & [58] & http://treegraph.bioinfweb.info/ \\
\hline R software & http://r-project.org & $\mathrm{N} / \mathrm{A}$ \\
\hline
\end{tabular}

\section{CONTACT FOR REAGENT AND RESOURCE SHARING}

Further information and requests for resources and reagents should be directed to and will be fulfilled by the Lead Contact, Fernanda de Carvalho-Niebel (fernanda.de-carvalho-niebel@inra.fr).

\section{EXPERIMENTAL MODEL AND SUBJECT DETAILS}

\section{Plant material, bacterial strains and growth conditions}

Medicago truncatula A17 wild-type and a derived transgenic line harboring the pEXPA:GFP construct (in the destination vector pBGW7) were used in this study. The pEXPA:GFP homozygous seed population used here (propagated for four generations) is derived from three independent transgenic lines that were generated and selected before by M. Smoker at the Sainsbury Lab (Norwich) [59], on the basis of their transgene copy number and GFP expression. M. truncatula seeds were scarified with sulfuric acid for $10 \mathrm{mn}$, rinsed with sterile water, and surface-sterilization in bleach water (12\% sodium hypochlorite) before incubation for 20 minutes in water. Sterilized seeds were germinated on inverted Campbell agar plates for $3-4$ days at $4{ }^{\circ} \mathrm{C}$ and overnight at $20^{\circ} \mathrm{C}-25^{\circ} \mathrm{C}$ (for further information, http://www.noble.org/medicagohandbook), before being transferred to various growth conditions for nodulation studies or used for $A$. rhizogenes transformation as described in the Method details below. The Escherichia coli strain DH5 $\alpha$ was used to propagate plasmid DNAs and the A. rhizogenes ARquA1 strain [51] was used for $A$. rhizogenes-mediated transformation. The following S. meliloti strains were used for rhizobia-inoculation experiments: the strain 1021 carrying a pXLGD4 lacZ reporter (Sm 1021-lacZ) [47], was used to inoculate transgenic roots expressing MtBG2/PdBG1 and in callose immunostaining assays; the strain 2011 constitutively expressing a hemA-lacZ fusion (Sm 2011-lacZ) [52], was used to inoculate aeroponically-grown seedlings or transgenic roots expressing pMtBG2:GUS, pNIN:GUS, pNF-YA1:GUS, pERN1:GUS, pERN1:MtBG2 RNAi or the cals3m/GFP constructs; the strain 2011 constitutively expressing CFP (Sm 2011-CFP) [53] was used in pEXPA:YFP symplastic flux inoculation experiments, and the strain 2011 constitutively expressing the mCherry fluorescent protein (Sm 2011-mCherry, kindly provided by J. Fournier, LIPM), was used in CFDA spot-inoculation experiments. E. coli strains were propagated at $37^{\circ} \mathrm{C}$ in Luria Bertani (LB) media while A. rhizogenes and S. meliloti strains were propagated at $28^{\circ} \mathrm{C}$ on selective tryptone yeast media supplemented with $6 \mathrm{mmol} . \mathrm{L}^{-1}$ calcium chloride and respective antibiotics. The selective growth of the $S$. meliloti strains was done in the presence of $10 \mu \mathrm{g} \cdot \mathrm{mL}^{-1}$ tetracycline. The growth of the $A$. rhizogenes ARquA1 strains carrying different binary vectors with various 
antibiotic resistances was done in the presence of $100 \mu \mathrm{g} \cdot \mathrm{mL}^{-1}$ streptomycin (helper plasmid resistance) and supplemental binary vector resistance antibiotics $\left(50 \mu \mathrm{g} \cdot \mathrm{mL}^{-1}\right.$ of kanamycin, $300 \mu \mathrm{g} \cdot \mathrm{mL}^{-1}$ of spectinomycin or $200 \mu \mathrm{g} \cdot \mathrm{mL}^{-1}$ chloramphenicol).

\section{DNA Constructs}

Promoters, cDNA sequences and fluorescent fusion proteins necessary for the generation of PdBG1 fusion proteins were synthesized by GenScript. For the PdBG1(At3g13560)-eGFP fusion, DNA fragments were concatenated using Golden-Gate assembly reactions [60] to generate the final fusion protein which expression is driven by pERN1 [18]. Cassettes to express DS-Red as a marker for transformation was also introduced in the binary vectors. For $p U B I Q-M t B G 2$ (labeled with mCherry), multisite Gateway cloning was used. In brief, primers were designed to amplify Medtr3g083580 (MtBG2), pUBIQ (isolated from AtUBIQ10) and mCherry (Table S2). C-terminal and N-terminal parts of the protein were amplified separately and cloned into different donor vectors by BP reaction. Plasmids from confirmed positive pDNR clones were used for LR reaction into destination vectors following manufacture's instruction (invitrogen) and transformed into $E$. coli. Colonies were screened by colony PCR and confirmed by sequencing. Following the same protocol, a version without mCherry fusion was generated. The transcriptional reporter $p M t B G 2: G U S, p N I N: G U S, p E R N 1$ : GUS, the pEXPA:PV $c$ ( $P V_{c d}$, parvalbumin mutated in EF hand motif, Addgene, reference Plasmid \#16345):NLS:CFP [54], pEXPA:YFP, pEXPA:mCherry-ER and the pERN1:MtBG2 RNAi constructs were obtained by Golden-Gate cloning in pCambiaCR1 derived vectors [55]. DNA fragments corresponding to $1400 \mathrm{bp}$ or $2100 \mathrm{bp}$ upstream the ATG of NIN, $1705 \mathrm{bp}$ upstream the ATG of MtBG2 (Medtr3g083580), 2200 bp upstream the ATG of ERN1, 401 bp of upstream EXPA sequences [30] were amplified by $\mathrm{PCR}$, cloned in pbluescript vectors and sequenced before concatenated using Golden-Gate assembly reactions [55]. The $p E R N 1$ : cals3m, pMtBG2:cals3m and $p A N N 1: c a l s 3 m$ constructs were generated by combining restriction enzyme and gateway cloning. First pMtERN1 (1926 bp from ATG), pMtBG2 (1705 bp from ATG) and pANN1 (2234 bp from ATG) DNA fragments were amplified by PCR using primers listed in Table S2, and sub-cloned into pBlueScript II. After sequencing validation and restriction digestion, pMtERN1 and $p A N N 1$ DNA fragments were inserted into HindlII and Spel sites of the pK7WG2-R-pLjUb vector [30], replacing 431 bp of the $L j U b$ promoter, and cloned just upstream the proximal 100bp sequences of pLjUb without regulatory sequences. The $p M t B G 2 \mathrm{DNA}$ fragment was inserted into HindlII and Spel sites of the pK7WG2-R-pEXPA vector replacing the $p E X P A$ promoter sequence [30]. The created pK7WG2-R-pMtERN1, pK7WG2-R-pANN1, pK7WG2-R-pMtBG2 constructs and the pK7WG2-R-pLjUb [30] and pK7WG2-R-pEXPA [30] vectors were used in LR gateway reactions with pDONR221-cals3m (kindly provided by Ykä Helariutta) and a pENTRY-GFP to create $p E R N 1: c a l s 3 m, p E R N 1: G F P, p A N N 1: c a l s 3 m, p A N N 1: G F P, p M t B G 2: c a l s 3 m, p M t B G 2: G F P, p L j U b$ : cals3m, $p E X P A: c a l s 3 m$, and $p E X P A: G F P$ constructs. The MtBG2-RNAi $5^{\prime}$ and $R N A i-c d s$ constructs were generated by using respectively the $250 \mathrm{bp}$ non-coding sequences upstream the ATG (5') or the $850 \mathrm{bp}$ (coding) sequences downstream the ATG of MtBG2 (cds). MtBG2 5'and cds sequences and the 1300 bp intron spacer sequence of the pK7GWIWG2(II)-RedRoot vector [56] were amplified by PCR and sequence validated before used for generated the RNAi constructs. MtBG2 5 'and cds sequences were assembled by Golden-Gate in the pCambiaCR1 [55] vector in sense and antisense orientations before and after the intron spacer sequence.

\section{METHOD DETAILS}

Generation and selection of $M$. truncatula transgenic roots

A. rhizogenes transformation of $M$. truncatula roots was done according to previous protocols [13, 61] with minor modifications. Germinated seedlings with a radicle length of approximately $10 \mathrm{~mm}$, were sectioned $3 \mathrm{~mm}$ from the root tip and placed on

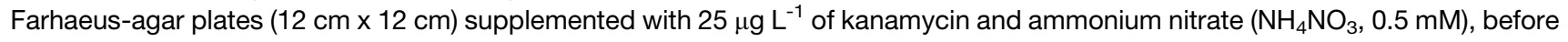
inoculation with a drop $(\sim 3 \mu \mathrm{l})$ of the $A$. rhizogenes bacterial suspension $\left(O D_{600 \mathrm{~nm}} 1\right)$. Plates were partially sealed with parafilm to allow crucial gas exchanges and placed vertically in plastic boxes at controlled environments with $16 \mathrm{~h}$ light/ $8 \mathrm{~h}$ dark photoperiods, at $20^{\circ} \mathrm{C}$ for 1 week and then at $25^{\circ} \mathrm{C}$ for two to three weeks before selection. Kanamycin resistant composite M. truncatula plants were selected under the stereomicroscope for the expression of the DsRED fluorescent protein encoded in the T-DNA of the transformation binary vectors. Individual DsRED ${ }^{+}$composite plants were transferred to pouch paper/Fahraeus agar plates (nitrogen and antibiotic free) [62] and used for S. meliloti inoculation as described below.

\section{Growth conditions and inoculation of M. truncatula roots}

For MtBG2/MtBG5 quantitative RT-PCR experiments, germinated $M$. truncatula A17 seedlings were directly transferred to aeroponic conditions [18] and nitrogen starved for 3 days before inoculation with a $S m$ 2011-lacZ suspension in water (OD 6000.002$)$. For immunolocalization, germinated seedlings were transferred to square plates containing Buffered Nodulation Medium [47] (BNM) supplemented with $1 \mu \mathrm{M} \mathrm{L}$ - $\alpha$-(2-aminoethoxyvinyl)-Gly (AVG) and $M$. truncatula roots were spot-inoculated with $2 \mu \mathrm{L}$ of a Sm 1021 -lacZ bacterial suspension in water $\left(\mathrm{OD}_{600}\right.$ of 0.002$)$ in the region of root hair emergence. For CFDA experiments, germinated seedlings were grown for 7-10 days in pouch paper/Fahraeus agar [18] plates before spot inoculation with $2 \mu \mathrm{L}$ of a Sm 2011$m$ Cherry bacterial suspension in water $\left(\mathrm{OD}_{600} 0.01\right)$ in the nodulation competence root zone. For YFP symplastic assays, composite plants were grown and flood inoculated with a water suspension of $S m$ 2011-CFP $\left(\mathrm{OD}_{600} 0.001\right)$. Similarly for the analysis of GFP transport in pEXPA-GFP transgenic roots, composite plants were grown for 3 weeks before flood inoculation with Sm 1021-lacZ bacterial suspension in water $\left(\mathrm{OD}_{600}\right.$ of 0.002$)$. For phenotyping transgenic roots expressing MtBG2/PdBG1 constructs, composite plants were transferred to either square plates containing BNM (with $1 \mu \mathrm{M}$ AVG) or a mixture of equal amounts of sand and terra green 4 weeks after transformation and were flooded with a suspension of $S m$ 1021-lacZ $\left(\mathrm{OD}_{600}\right.$ of 0.05$)$ in $10 \mathrm{mM} \mathrm{MgCl}_{2}$. Transgenic roots 
expressing pMtBG2:GUS, pMtNIN:GUS, pMtNF-YA1:GUS [42], pERN1:GUS, pERN1:MtBG2 RNAi and GFP/cals3m constructs driven by $p E X P A, p E R N 1, p M t B G 2$ or $p A N N 1$ promoters were transferred to pouch paper/Fahraeus agar plates 2-4 weeks posttransformation and flood inoculated with a Sm 2011-lacZ suspension $\left(\mathrm{OD}_{600}\right.$ of 0.01$)$ in water. After $1 \mathrm{~h}$, excess solutions were removed and plates with composite plants were incubated vertically until sample harvesting. Non-inoculated or mock (water) inoculated controls were used in all experiments listed above. After transferring to respective growth conditions, plates with composite plants were randomly divided in two pools, to be subsequently inoculated and processed as control or rhizobia-inoculated samples. Biological experiments involved the analyses of root samples of individual plants issued from independent biological experiments. The number of samples analyzed $(n)$, and the number of biological repetitions are indicated in the Figure legends for each experiment. Individual root systems of composite plants were subsequently used for in vivo imaging, histochemical GUS/ $\beta$-galactosidase staining followed by microscopy analyses or ground in liquid nitrogen for RNA extraction and qRT-PCR, 1-16 days post inoculation.

\section{GUS and $\beta$-Galactosidase assays}

Inoculated or non-inoculated root samples of composite $M$. truncatula plants were prefixed in $0.5 \%$ paraformaldehyde/0.1 M potassium phosphate buffer $\mathrm{pH} 7.0$, for $1 \mathrm{~h}$, before histochemical (blue) staining for GUS activity was performed for $2-5$ hours at $37^{\circ} \mathrm{C}$ using $1 \mathrm{mM}$ of the substrate X-Gluc (5-bromo-4-chloro-3-indoxyl-b-D-GlcA, cyclohexylammonium salt, B7300; Biosynth, Staad, Switzerland) $[10,11]$. To reveal the constitutive $\beta$-galactosidase activity of S. meliloti strains Sm 2011-lacZ and Sm 1021-lacZ in inoculated root tissues, GUS-stained or non-stained root samples were rinsed in $Z$ buffer $(10 \mathrm{mM} \mathrm{KCl,} 1 \mathrm{mM} \mathrm{MgCl} 2$, and $0.1 \mathrm{M}$ phosphate buffer, $\mathrm{pH} 7.4$ ) and fixed for $1 \mathrm{~h}$ in $1.25 \%$ glutaraldehyde/ $\mathrm{Z}$ buffer. After $Z$ buffer rinsing, root samples were incubated overnight in the dark at $28^{\circ} \mathrm{C}$ in Z buffer containing $2 \mathrm{mM}$ of the Magenta-Gal (5-bromo-6-chloro-3-indoxyl-b-D-galactopyranoside; B7200; Biosynth) or the blue X-Gal (5-bromo-4-chloro-3-indolyl-b-Dgalactopyranoside,W5376C; Thermo Fisher Scientific, Guilford, CT) substrates $[22,47]$. GUS, GUS/LacZ or LacZ stained tissues were occasionally cleared for $30 \mathrm{~s}$ with $12 \%$ sodium hypochlorite solution before microscopy observations, or collected for Technovit 7100 embedding as described below.

\section{Symplastic transport assays}

Carboxy Fluorescein Diacetate (CFDA, Sigma) was dissolved in DMSO $(24 \mathrm{mg} / \mathrm{ml})$ and stored at $-20^{\circ} \mathrm{C}$. Plants were grown on pouch paper/Fahraeus agar plates [18] for 7-10 days, before being spot inoculated with the $S m$ 2011-mCherry strain $\left(O D_{600} 0.01\right)$. A freshly prepared CFDA working solution $(60 \mu \mathrm{g} / \mathrm{ml})$ in $\mathrm{H}_{2} \mathrm{O}$ was added to the roots. After 20 min incubation in the dark, roots were rinsed at least twice with water and used for microscopy 2-3 days post-inoculation. Composite transgenic roots expressing $P E X P A-Y F P$ and pEXPA:mCherry-ER constructs were grown on pouch paper/Fahraeus agar plates and inoculated with the Sm 2011-CFP strain $\left(\mathrm{OD}_{600} 0.001\right)$ and analyzed by confocal microscopy 2-3 days post-inoculation. Data were obtained from 2-3 biological experiments after analyses of 20 plants. $p E X P A: G F P$ stable transgenic lines transformed via $A$. rhizogenes with $p E R N 1: c a l s 3 m, p U B I Q-M t B G 2$ or an empty vector control were grown for 3 weeks in Fahraeus agar plates and rhizobia-inoculated as described above. GFP expression was visualized in three independent transformation events under confocal microscopy 5 days post-inoculation and images analyzed as described in the Microscopy methods section.

\section{Callose immunolocalization}

M. truncatula roots were spot-inoculated with the Sm 1021-lacZ strain as described above. Root sections were selected $0.5 \mathrm{~cm}$ above and below the region where roots hairs start to emerge, as this zone appears the most susceptible to infection by spot-inoculation. The selected root zone was sectioned into $1-2 \mathrm{~cm}$ pieces and fixed in 4\% paraformaldehyde in PBS buffer (pH 7.0) supplemented with $0.01 \%$ Triton-100 before being dehydrated in ascending ethanol:water series. Samples were stained with a $2 \%$ eosin solution to facilitate sample positioning during embedding and sectioning as described in [63] with the following modifications: wax changes were performed for 5 days and blocks were stored at $-20^{\circ} \mathrm{C}$ until they were processed. Sections of $10 \mu \mathrm{m}$ were collected using a Rotatory Microtome HM 325 (MICROM) in polylysine slides (VWR International). Slides were kept at $-20^{\circ} \mathrm{C}$ overnight before following steps. Callose immunolocalization was performed using a monoclonal antibody (Biosupplies, http://www. biosupplies.com. au/) as described [63]. Briefly, after removal of wax, sections were treated with $0.1 \mathrm{M} \mathrm{NH}_{4} \mathrm{Cl}$ (in PBS) for 5 min, blocked with $5 \%$ BSA and exposed to the primary antibody O/N. Detection was carried out using Alexa Fluor ${ }^{\circledR} 488$ anti-mouse (Invitrogen). All steps were carried out at room temperature unless stated otherwise. When hand sections were needed, roots were sectioned with a razor blade, exposing the inner tissue, and were let drying for two hours at room temperature before immunolocalization.

\section{Microscopy methods}

Aniline blue was used to stain callose at the cell wall in entire roots. Roots were cut and rapidly submerged in $0.01 \%$ (w/v) aniline blue in phosphate buffer ( $\mathrm{pH} 9$ ), vacuum infiltrated for $15 \mathrm{~min}$ and incubated overnight in the dark at RT. Roots were rinsed in water before imaging. Confocal analysis was performed on a Zeiss LSM700 Inverted and LSM800 upright microscope using a $488 \mathrm{~nm}$ excitation laser for GFP or a $405 \mathrm{~nm}$ for CFP/aniline blue staining. Emission was collected using the filters: BP 505-530 for GFP and the DAPI filter for aniline blue $(463 \mathrm{~nm})$. The images corresponded to individual stacks of z-optical sections. For quantification, confocal images of callose immunolocalization in $M$. truncatula roots were taken under non-saturated conditions and regions of interest (ROI) of $100 \mu \mathrm{m}^{2}$ were drawn in developmentally comparable areas of spot-inoculated roots comprising pericycle/endodermis and inner cortex. Fluorescence intensity analysis was performed using ImageJ software (http://imagej.nih.gov/ij) [57]. The integrated density was corrected for background fluorescence by dividing the measured intensities with the average intensity of a cell-free (dark) region. For 
each condition, the fluorescence intensity of 3 individual roots was analyzed. Similar protocols were followed for quantification of GFP fluorescence in images taken in the pEXPA-GFP background. In this case, mean gray values for each image were calculated in more than 5 independent $\mathrm{ROI}$ of $55 \mu \mathrm{m}$ wide at different distances from the epidermis (as represented in the $\mathrm{x}$ axis of the graphs).

Analyse of CFDA/GFP symplastic conductivity were carried out by monitoring CFDA, YFP, mCherry-ER and the bacterial CFP fluorescence signals by confocal microscopy using a Leica SP8 laser point scanning microscope. The argon laser bands of 458 and $488 \mathrm{~nm}$ were used to excite CFP, and YFP/CFDA, respectively, and a 561-nm diode to excite the mCherry. Specific emission windows used for CFP, YFP, mCherry were 465 to $485 \mathrm{~nm}, 500$ to $550 \mathrm{~nm}, 600$ to $630 \mathrm{~nm}$. The images shown are single confocal sections, maximal projections of selected planes of a z stack. Images were acquired and projected and processed using Leica, ImageJ (http://imagej.nih.gov/ij/) [57] or Volocity version 6.0.1 (PerkinElmer) softwares.

Longitudinal $(8-10 \mu \mathrm{m})$ sections were generated for the detailed phenotypic analyses of $p M t B G 2: G F P / c a l s 3 m$ and $p E R N 1: G F P /$ cals3m rhizobia-inoculated root or nodule tissues and for the the tissue-specific analysis of $p M t B G 2: G U S, p N I N: G U S$ and $p N F-Y A 1$ : GUS expression (with or without GFP or cals $3 \mathrm{~m}$ expression). Briefly, GUS/lacZ or lacZ-stained root tissues collected at 7 or $12 \mathrm{dpi}$ were cut in $\sim 1 \mathrm{~cm}$ root pieces and fixed in 1.5\% glutaraldehyde for 1 hour, re-rinsed and sequentially dehydrated using an ethanol series (from $30 \%, 50 \%, 70 \%, 90 \%$ to $100 \%$ ethanol for $60 \mathrm{~min}$ each). Subsequently, tissues were embedded in ethanol:Technovit ${ }^{\circledR} 7100$ (Heraeus-Kulzer, Germany) LP series (3/1, 2/1, 1/1,1/2, 1/3 v/v, each step 60 min) before an overnight incubation in $100 \%$ Technovit ${ }^{\circledR} 7100$ LP. Samples within the final Technovit polymerization solution were left to polymerize overnight. Each Technovit ${ }^{\circledR} 7100$ block comprised 4-5 root pieces of independent composite plants, each comprising variable numbers of nodule primordia or nodule structures, depending of whether it was a control or a cals $3 \mathrm{~m}$ sample. 5 blocks (with $\sim 20$ root pieces of independent plants) were cut for pMtBG2:cals3m, pERN1:cals3m/pNIN:GUS and respective pMtBG2:GFP and pNIN:GUS control samples. 4 blocks (with 16 root pieces of independent plants) and 1 block (with 5 root pieces of independent plants) were respectively cut for $p E R N 1: c a l s 3 m / p N F-Y A 1: G U S$ and $p N F-Y A 1: G U S$ control samples. 15 blocks (with 60 root pieces of independent plants) were cut for $p E R N 1: c a l s 3 m$ and respective $p E R N 1: G F P$ or non-transformed control samples. Technovit blocs were sectioned to 8-10 $\mu \mathrm{m}$ by using an Ultracut E ultramicrotome (Reichert-Jung, Germany). Thin sections of one bloc were distributed on 4-6 slides and stained with Ruthenium Red (4-5 min, $0,05 \%$ in $\mathrm{H}_{2} \mathrm{O}$ ) to reveal cell outlines. Slides with poor quality sections and/or not cutting in the longitudinal axis were not considered for further microscopic analyses. Visualization of GUS or lacZ-staining tissues was done using a stereomicroscope (Leica Microsystems, Wetzlar, Germany), light microscopes (an AxioPlan 2 Imaging; Carl Zeiss, Oberkochen, Germany and a GX L1500light microscope), and/or CCD cameras (AxioCam MRc; Carl Zeiss Olympus -BH2 fitted with a QIMAGING camera, Canada). Images taken from individual root, nodule primordia or nodule sections were subsequently adjusted (luminosity, contrast or focus) by using ImageJ or Powerpoint softwares. Sections shown in Figures 4 and 5 were obtained after the analyses of $9,16,28$, and 15 individual sections of $p M t B G 2: G F P, p M t B G 2: c a l s 3 m$, control (pERN1:GFP and non-transformed plants) or pERN1:cals3m, respectively, from 2 experiments.

\section{RNA extraction and QRT-PCR analyses}

The entire root system of aeroponic grown A17 plantlets (for MtBG2/MtBG5 expression analyses) or individual composite plants (used in transgenic ectopic and GFP/cals3m experiments) were used for RNA extraction. Total RNA was extracted from M. truncatula roots using the Macherey-Nagel total RNA isolation kit according to the manufacturer's instructions. The DNA-free RNA samples were quantified, and RNA integrity was checked by Agilent RNA Nano Chip (Agilent Technologies). First-strand complementary DNA synthesis was performed using $1 \mu \mathrm{g}$ of total RNA with an anchored oligo (dT) and Transcriptor Reverse Transcriptase (Roche) following the manufacturers' protocol. Quantitative RT-PCR was performed on 384-well plates using the Light Cycler 480 system (Roche) and the SYBR Green I Master mix (Roche), according to the manufacturer's instructions. Each reaction was performed with $2 \mu \mathrm{L}$ of 1:20 (v/v) dilution of the first complementary DNA strand, with $0.5 \mu \mathrm{M}$ of each primer in a total reaction volume of $7 \mu \mathrm{L}$. Primer pairs to amplify MtBG2, MtBG5, GFP, NPL1, cals3m and CRE1 transcripts are listed in Table S2. Those used to amplify ERN1, ENOD11, NIN, Ubiquitin, NFYA1, Mt0089_00067, Mt0085_00020, MtCLE12 and MtCLE13 were previously described $[18,22,32,42]$. The PCR cycling conditions were as follows: $95^{\circ} \mathrm{C}$ for $5 \mathrm{~min}$ followed by 45 cycles of denaturation at $95^{\circ} \mathrm{C}$ for $15 \mathrm{~s}$, annealing at $60^{\circ} \mathrm{C}$ for $20 \mathrm{~s}$, and extension at $72^{\circ} \mathrm{C}$ for $20 \mathrm{~s}$. PCR amplification specificity was verified by analysis of the dissociation curve at the end of the PCR cycles by heating samples from $65^{\circ} \mathrm{C}$ to $95^{\circ} \mathrm{C}$. Only samples showing a clear unique melting peak in the expected temperature range were considered for further analysis. To confirm the specificity of the primer pairs, we purified the amplified PCR products from the QRT-PCR reactions using the Promega Wizard kit, and used them directly of after subcloning for DNA sequencing. The MtBG2/MtBG5 genes were selected for Q-RT-PCR analyses following their in silico expression profiles in rhizobial root hair infectome [47] and/or laser-dissected nodule zones [64] transcriptomes. For the analyses of symbiotic gene expression in pERN1:GFP or pERN1:cals3m and RNAi roots, we first analyzed by Q-RT-PCR the expression of GFP or calS3m in individual root samples. Only $\mathrm{GFP}^{+}$and calS3m $\mathrm{m}^{+}$samples were considered for the further analyses of symbiotic gene expression as shown in Figures 6 and S6. The Q-RT-PCR data represent mean values of five (Figures 11 and S1C), three (Figures 6A-6D) or two (Figures S6A-S6P) independent biological experiments, with at least two technical replicates after normalization with the three reference transcripts (Ubiquitin, Mt0089_00067, and Mt0085_00020) shown before to exhibit invariable expression levels [18].

\section{Phylogenetic analysis}

The selection of $M$. truncatula and $A$. thaliana sequences containing the $\beta$-1,3-glucosidase domain (GHL17) and other characteristic features of this family: a signal peptide (SP), a glycosyl phosphatidylinositol anchor (GPI) and a carbohydrate-binding module (X8) 
was done using prediction tools as described [39]. Only protein sequences containing a complete GHL17 domain were used for phylogenetic trees calculations as described in [39]. The best model under the Akaike Information Criterion was LG+G. The Majority-rule consensus trees convergence was reached after 1130000 generations. The trees were visualized using Figtree (http://tree. bio.ed.ac.uk/software/figtree/) and edited using TreeGraph2 [58]. Note that Figures 1 and S1 only shows the Bayesian tree, the other Maximum Likelihood and Neighboring-Joining trees are available on request.

\section{QUANTIFICATION AND STATISTICAL ANALYSIS}

Data was obtained from independent biological replicates (and technical replicates in the case of QRT-PCR analyses) and after the analyses of individual randomly distributed samples. The number of individually analyzed samples (n), number of replicates and $p$, significant level values are indicated in Figure legends. All statistical analyses were performed using the R software (http:// r-project.org). Data were evaluated for normality using a Shapiro-Wilk test and for homogeneity of variances using both Fisher and Bartlett tests. Parametric (ANOVA and two sample t test) or non-parametric (Mann-Whitney and Kruskal-Wallis) statistical tests were used for normally-distributed or non-normally distributed samples, respectively. Integrated density fluorescent signal values in Figure $1 \mathrm{G}$ and ERN1 QRT-PCR data in Figure 6A, showing a normal distribution but heterogeneity of the variance, were analyzed using the Aspin-Welch two sample t test (for data in Figure 1G, T = -4.6349, $\mathrm{p}<0.001$ and for the ERN1 QRT-PCR data in Figure 6A: $\mathrm{T}=-2.0765, \mathrm{p}<0.05)$. Root weight data in Figure S5C were first transformed into normality using Log ${ }_{10}$ data values before statistical analysis using the Aspin-Welch two sample t test ( $\mathrm{T}=-1.8763, \mathrm{p}>0.05)$. MtBG2 and MtBG5 QRT-PCR data (Figures 1 I and S1C) were normally-distributed and showed homogeneity of variances, thus statistical analyses of the data was done using one-way ANOVA followed by Tukey honest significant difference (HSD) tests (For MtBG2: $F=9.618, p<0.001$; for MtBG5: $F=14.25$, $\mathrm{p}$ < 0.001). CRE1 (3 and $7 \mathrm{dpi}$ ), CLE12 (3 dpi) and CLE13 (3 dpi) QRT-PCR data in Figure S6 were first transformed into normality using Box-Cox (S6E $\lambda=-1.10, \mathrm{~S} 6 \mathrm{~F} \lambda=-0.06, \mathrm{~S} 6 \mathrm{G} \lambda=1.31, \mathrm{~S} 6 \mathrm{O} \lambda=1.6)$ before statistical analysis using one-way ANOVA followed by Tukey honest significant difference (HSD) tests $(F=0.631, p>0.05$ for Figure S6E; $F=4.555, p<0.01$ for Figure S6F; $F=0.221$, $\mathrm{p}>0.05$ for Figure S6G; $F=2.974, p<0.05$ for Figure S6O). MtBG2 QRT-PCR data in Figure S5B were first transformed into normality using Box-Cox $(\lambda=0.67)$ before statistical analysis using one-way ANOVA followed by Tukey honest significant difference (HSD) tests $(F=0.030538, p<0.001)$. Integrated density fluorescent signal values in Figure S2Q and ENOD11, NIN and NFYA1 QRT-PCR data in Figure 6 were first transformed into normality using Box-Cox (Figure S2Q $\lambda=0.14$ and Figure $6 \mathrm{~B} \lambda=0.22$ ) or Log ${ }_{10}$ data values (Figures $6 C$ and $6 D)$, before statistical analysis using a Two sample t test $(T=-4.3291, p<0.001$ for Figure $S 2 Q ; T=1.608, p>0.05$ for Figure 6B; $\mathrm{T}=3.8477, \mathrm{p}<0.001$ for Figure $6 \mathrm{C} ; \mathrm{T}=4.5582, \mathrm{p}<0.001$ for Figure $6 \mathrm{D}$ ). The nodule number data in Figure S4B, following a normal distribution and homogeneity of the variance were analyzed using a two-sample t test $(T=1.4701, p>0.05)$. Data in Figures 3G-3I, 4A-4C, 5A-5C, S4A, and S5F-S5H did not follow a normal distribution and were then analyzed using the non parametrical Mann-Whitney test: Figures 3G-3I (3G, $W=129.5, p<0.001$; $3 \mathrm{H}, \mathrm{W}=149, \mathrm{p}<0.001$; and $3 \mathrm{I}, \mathrm{W}=170, \mathrm{p}<0.001)$; Figures 4A-4C $(4 \mathrm{~A}, \mathrm{~W}=1210, \mathrm{p}<0.05 ; 4 \mathrm{~B}, \mathrm{~W}=1169.5, \mathrm{p}<0.05 ; 4 \mathrm{C}, \mathrm{W}=1754.5 \mathrm{p}>0.05)$; Figures $5 \mathrm{~A}-5 \mathrm{C}(5 \mathrm{~A}, \mathrm{~W}=3995.5, \mathrm{p}<0.001 ; 5 \mathrm{~B}, \mathrm{~W}=$ 4232.5, $p<0.001 ; 5 C, W=1489, p<0.001)$; Figure S4A (W = 740, p < 0.001) and Figures S5G-S5I (S5F, W = 78, p < 0.01; S5G, $\mathrm{W}=82.5, \mathrm{p}<0.01 ; \mathrm{S} 5 \mathrm{H}, \mathrm{W}=935.5, \mathrm{p}<0.05)$.

Data in Figures 4L-4N, S5A, S6A-S6D, S6H-S6N, and S6P did not follow a normal distribution and equality of the variance and where then analyzed using the non parametrical Kruskal-Wallis test $(4 \mathrm{~L}$, Chisq $=21.47, p<0.001 ; 4 \mathrm{M}, \mathrm{Chisq}=21.83, \mathrm{p}<0.001$; $4 \mathrm{~N}$, Chisq $=10.8, \mathrm{p}<0.01$; S5A, Chisq $=8.01, \mathrm{p}<0.05$; S6A, Chisq $=44.73, \mathrm{p}<0.001$; S6B, Chisq $=26.56, \mathrm{p}<0.001 ; \mathrm{S} 6 \mathrm{C}, \mathrm{Chisq}=$ 42.96, $\mathrm{p}<0.001$; S6D, Chisq $=52.44, \mathrm{p}<0.001$; S6H, Chisq $=46.46, \mathrm{p}<0.001$; S6I, Chisq $=46.27, \mathrm{p}<0.001$; S6J, Chisq $=32.97$, $\mathrm{p}<0.001$; S6K, Chisq $=43.78, \mathrm{p}<0.001$; S6L, Chisq $=37.02, \mathrm{p}<0.001$; S6M, Chisq $=21.99, \mathrm{p}<0.001$; S6N, Chisq $=28.97, \mathrm{p}<0.001$; $\mathrm{S} 6 \mathrm{P}$, Chisq $=31.79, \mathrm{p}<0.001)$.

\section{DATA AND SOFTWARE AVAILABILITY}

The raw and analyzed data reported in this paper is available on the Mendeley database at https://doi.org/10.17632/5hzcxdb4dp.1. 